\title{
Enhancing the Performance of Intelligent Control Systems in the Face of Higher Levels of Complexity and Uncertainty
}

\author{
Randa Herzallah \\ Faculty of Engineering Technology \\ Al-Balqa' Applied University, Jordan \\ Email:randa_herzal@hotmail.com
}

\begin{abstract}
Modern advances in technology have led to more complex manufacturing processes whose success centers on the ability to control these processes with a very high level of accuracy. Plant complexity inevitably leads to poor models that exhibit a high degree of parametric or functional uncertainty. The situation becomes even more complex if the plant to be controlled is characterized by a multi-valued function or even if it exhibits a number of modes of behavior during its operation. Since an intelligent controller is expected to operate and guarantee the best performance where complexity and uncertainty coexist and interact, control engineers and theorists have recently developed new control techniques under the framework of intelligent control to enhance the performance of the controller for more complex and uncertain plants. These techniques are based on incorporating model uncertainty. The new developed control algorithms for incorporating model uncertainty are proven to give more accurate control results under uncertain conditions. In this paper we survey some approaches that appear to be promising for enhancing the performance of intelligent control systems in the face of higher levels of complexity and uncertainty.
\end{abstract}

\section{INTRODUCTION}

In control problems there are degrees of uncertainty with respect to the process to be controlled. In general, uncertainty in control problems arises because of insufficient knowledge about the system itself or the environment in which it operates. This could be due to: the nondeterministic relationship between the input and the output variables of the system, or the system itself is too 
complex to represent, or unexpected changes in the system characteristics because of failure or time varying properties.

A large number of control techniques, including classical control, optimal control, and robust control have been developed to ensure that a system of interest performs according to predefined desired specifications under uncertain conditions. For example, the technique of adaptive control [2], [33], [43] has been shown capable of maintaining adequate performance in the presence of parametric uncertainty. Progress has been reported on the convergence, stability and robustness properties of linear adaptive control systems, both in discrete and continuous time [15], [47]. Techniques such as feedback linearization have been proposed for feedback linearizable nonlinear systems [28], [54]. Most of the progress on nonlinear adaptive control has been for continuous time systems. Progress in nonlinear discrete adaptive control systems has been slower and less general in scope [60].

Despite this progress, the theory of conventional adaptive control can hardly maintain good performance when sudden changes, such as sensor or actuator failures, occur in the process. In addition the theory deals only with parametric uncertain nonlinear systems where systems are usually characterized by known nonlinear functions and unknown constant parameters which are also required to appear linearly in the system equation. A more reliable adaptive control scheme stems from the theory of stochastic adaptive control [2], [58]. In stochastic adaptive control, the control law is derived by minimizing a performance index that usually takes the form of the expected value of some cost function. This implies that unlike the conventional adaptive control scheme, uncertainty of the parameters' estimate is taken into consideration in the control algorithm. This control law is referred to as dual control [11]-[13]. Unfortunately, dual control is shown to be computationally expensive and therefore has not been widely used in practice.

A universal method to deal with nonlinear stochastic or deterministic control systems is dynamic programming [35], [40], [52]. Several solutions for solving stochastic nonlinear control problems have been proposed in the literature [35], [40]. One of the proposed solutions to evaluate optimal control for stochastic systems [35] is to first find the best estimate of the state of the system from the measurements and then to apply the control that is optimal if the observed variable of the system is equal to this estimate. This however is practically infeasible, not least because of the unbounded search space which is needed to try and maintain all possible solution trajectories. 
The success of modern complex manufacturing processes centers on the ability to control these processes with a very high level of accuracy. Model uncertainty is usually inevitable when identifying highly complex systems. This is not simply because of the uncertainty arising from the estimated parameters or functions. It concerns also the structure and the complexity of the model and the appropriate choice of a cost function. For such complex systems, neural networks have often been regarded as the key solution for getting more accurate models and better generalization properties.

A pioneering paper in neural adaptive control was published by Narendra and Parthasarathy [48]. An adaptive neurocontroller with guaranteed stability was developed by Polycarpou and Ioannou [41] and Sanner and Soltine [53] for systems with no internal dynamics, whilst TzirkelHancock and Fallside [55] generalized this to systems having internal dynamics. More publications in the neuro-control field with different control algorithms can be found in [3], [9], [26], [36], [42], [61]. Almost all those papers developed control algorithms based on the assumption that the outputs of the neural networks are accurate. More precisely, the control law is derived based on the certainty equivalence principle.

Although neural networks have helped in estimating more accurate models, it has been shown that for most real world control problems with unpredictable disturbance and which exhibit a number of distinct modes of behavior during their operation, the predicted output of the neural network is inherently uncertain. So how could we proceed in such situations? The solution to this problem has been addressed from the point of view of estimating and incorporating uncertainty. It has been argued that for such complex systems, it may be the case that incorporating uncertainty in control design can help provide a better control result. Recently some publications have considered the use of knowledge of uncertainty to build a more robust controller.

Consequently new control algorithms are being developed for incorporating uncertainty in controller design. The purpose of this paper is to provide an overview of some of the recently developed control algorithms that have been reported in the literature concerning this subject. Mainly this paper concentrates on the recently developed control methods for incorporating uncertainty in the framework of indirect inverse control, adaptive control and adaptive critic methods. Figure 1 shows an overview of the three control methods. In addition, problems which need to be solved yet and future work will be identified. 


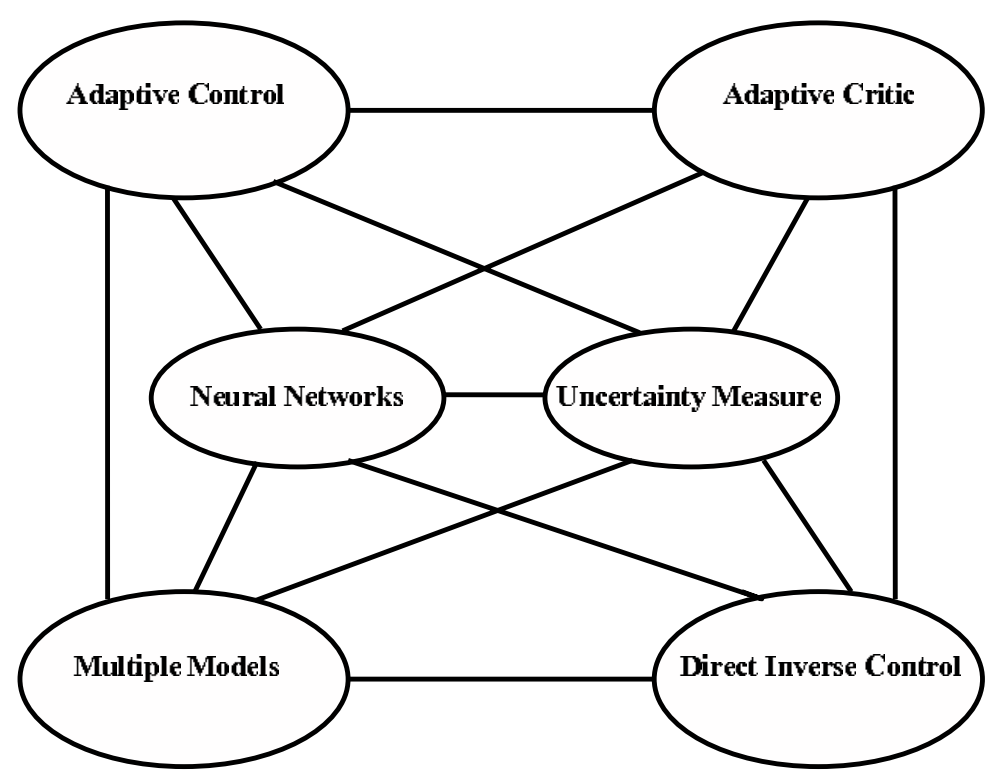

Fig. 1. Some of the control methods that are related to neural networks. It is usually assumed that neural networks can offer solutions for complex control systems. Neural network architectures are powerful because of nonlinearities, but uncertain because of incomplete knowledge and disturbances that are involved in control problems.

\section{INTELLIGENT CONTROL SYSTEMS}

In the neuro-control field a large number of publications describing the use of neural network models for control of linear and nonlinear systems have been published this decade. As a result a number of design approaches have been developed in the literature. Almost all these approaches assume the availability of an accurate mathematical model. This assumption however, is not reliable when speaking about complex nonlinear control problems which often also exhibit a number of distinct modes of behavior during their operation. The purpose of this section is to give an overview of some of the current available control techniques, which are derived based on the above assumption.

One of the neural network control schemes that is based on supervised learning methods and the assumption of an accurate model is the inverse control methodology [1], [27]. In the direct inverse control scheme [50], the inverse of the plant to be controlled is modeled off-line. Here the unity operator between the reference input and the output is approximated by connecting the plant inverse model network in series with the plant in an open loop configuration. This scheme however, is not very robust due to the absence of feedback. A more robust scheme 
that belongs to the inverse control methodology, utilizes a second neural network that has been trained previously to model the dynamics of the plant [26]. This is called internal model control (IMC).

Adaptive control techniques [2], [43] have been used in situations where the plant parameters are uncertain, because of the ability to maintain adequate performance in the presence of unknown or time varying parameters. Mainly, two methods have been reported in conventional adaptive control for handling adaptation: the direct and indirect methods [2], [43]. Concepts from conventional adaptive control theory have been extended naturally to the neural control case [48]. This is because the neural network models approximate nonlinear functions by a parameterized mapping. The paper by Narendra and Parthasarathy [48] is often considered as the pioneering paper in this field. It concentrated on discrete time systems and introduced four different classes of models. The paper dealt with adaptive control for nonlinear plants. It focused on indirect adaptive control because of the lack of methods for directly adjusting the parameters of the controller using only output error between the plant and the reference input.

A more complex approach than the previously mentioned methods, but more powerful under uncertain conditions is the adaptive critic family [39], [57]. This approach approximates dynamic programming which is one of the optimal methods for deriving the optimal control law for stochastic nonlinear control problems. Its application to real world nonlinear stochastic systems has been proven to be powerful and computationally feasible.

However, no neural control techniques can give good control results when the plant is operating in suddenly-changing environments or when it exhibits different features in different zones of its input. This type of plant complexity is called multi-modality [10] and usually is not accommodated for in the above mentioned neural control techniques. In conventional control methods, this type of plant complexity has been handled by the use of multiple models. The multiple model control approach has been developed from the partitioning theory of adaptive control [34]. The successful use of multiple models in real time applications for control has been widely reported in the literature [10], [16], [29], [30], [37], [44]-[46], [51]. Multiple model control techniques have been recently applied to the neural network models. Consequently, a number of multiple neural network methods such as the mixture of expert approach [6], [29], [31], [32], multiple paired forward and inverse models [59] and the mixture density network approach [21] have been developed. 


\section{INCORPORATING UNCERTAINTY}

In this section a discussion about how uncertainty knowledge is incorporated in the three neural control methods discussed in the previous section is provided. The next section will survey some of the multiple model approaches for incorporating uncertainty.

\section{A. Direct Inverse Adaptive Control}

In direct inverse adaptive control, the controller is learning to recreate the input that created the desired output of the plant [1], [4], [26], [50]. Here, the error for adapting the controller is the command error as shown in Figure 2. Using the command error for adapting the controller rather than trajectory error has several drawbacks [26], [50]. Mainly, the learning procedure is not goal directed, since in control minimizing the trajectory error (the difference between the system and the desired outputs) rather than command error is required. In addition, obtaining the inverse of the system may not be possible in problems where the mapping is not one-to-one.

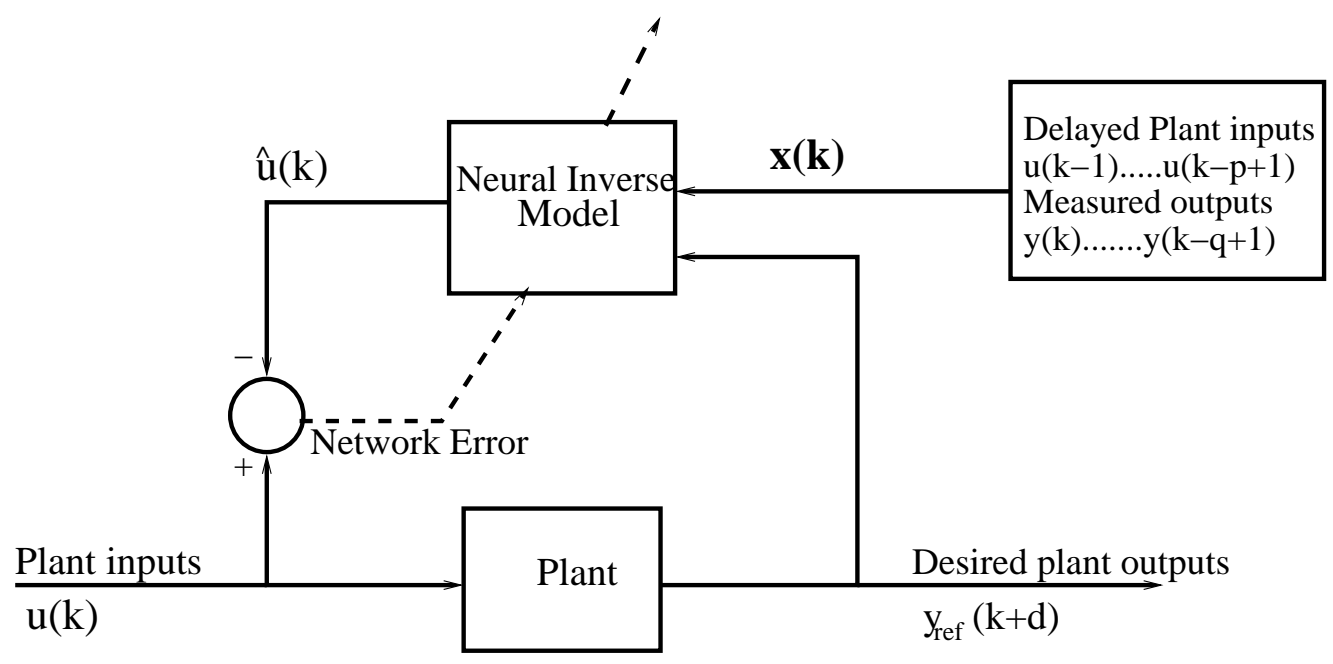

Fig. 2. Training of an inverse controller. Here the command error is used to adapt the parameters of the inverse controller.

To overcome these problems researchers considered the use of model uncertainty [20], [21]. In [21] a novel inversion-based neurocontroller for solving control problems involving uncertain nonlinear systems which could also compensate for multi-valued system (where the mapping is not one-to-one) is introduced. The approach is based on modeling the conditional distributions of both forward and inverse models. 
In their work [21] the conditional distributions of the residual error of the models are assumed to be Gaussian. The Gaussian assumptions for the residual errors of forward and inverse models are based on Theorem 4.2.1 in [14]. The theorem states that minimum mean squared error (MMSE) estimate of a random vector $y$ given another random vector $x$ is simply the conditional expectation of $y$ given $x, \hat{y}=E(y \mid x)$. It has also been stressed that the conditional expectation of $y$ given $x$ could be estimated using a nonlinear model. Based on this theorem the variance for each input pattern $x$ is shown to be given by $\|y-\hat{y}\|^{2}$, given that a good model could be estimated to approximate the random vector $\mathrm{y}$ given the random vector $x$ [21]. Another neural network, assumed to be nonlinear as well, which takes this variance as a target value has been used in [21] to model the conditional expectation of the variance of the residual error, $\sigma^{2}(x)=E\left(\|y-\hat{y}\|^{2} \mid x\right)$. In this way, the distribution of the random variable y could be described by a Gaussian function with an $x$ dependent mean and an $x$ dependent variance estimated by nonlinear models. This does not require the conditional distribution of the output variables to be Gaussian. If a sum of squares error is used, the quantities which can be determined are the x-dependent mean of the distribution (given by the output of the first trained neural network to predict the output variable) and the $x$-dependent variance (given by the output of the second trained neural network to predict the variance of the residual errors).

Rather than taking the conditional expectation from the inverse controller to represent the control signal to be forwarded to the plant, they suggested searching for the optimal control signal by generating samples from the conditional distribution of the inverse controller. Based on importance sampling from that distribution, the optimal control law is taken to be the one that minimizes the following performance index

$$
J(k)=\operatorname{Min}_{u \in U} E\left[\left(\hat{y}(k+d)-y_{\text {ref }}(k+d)\right)^{2}+\sigma_{\xi}^{2}\right]
$$

where $\mathrm{U}$ is a vector containing the sampled values from the control signal distribution, $\mathrm{E}$ is the expected value of the cost function over the random noise variable $v$, and $\sigma_{\xi}^{2}$ is the variance of the uncertainty of the forward model. The architecture of this control method is shown in Figure 3.

Generating samples from the conditional distribution of the inverse controller and then finding the control signal that minimizes a performance index of the form given in (1) makes the direct inverse control approach goal directed in terms of minimizing trajectory error rather than 
command error. Moreover, searching for the control signal that minimizes the performance index in (1) rather than using gradient information of (1) guarantees obtaining the absolute minimum of the performance index rather than a relative minimum [5]. Finally, for systems driven by a random forcing component the searching method allows approximating the integral of the utility function over the random variable, which is not easy to be done analytically, by the finite sum as given in (1).

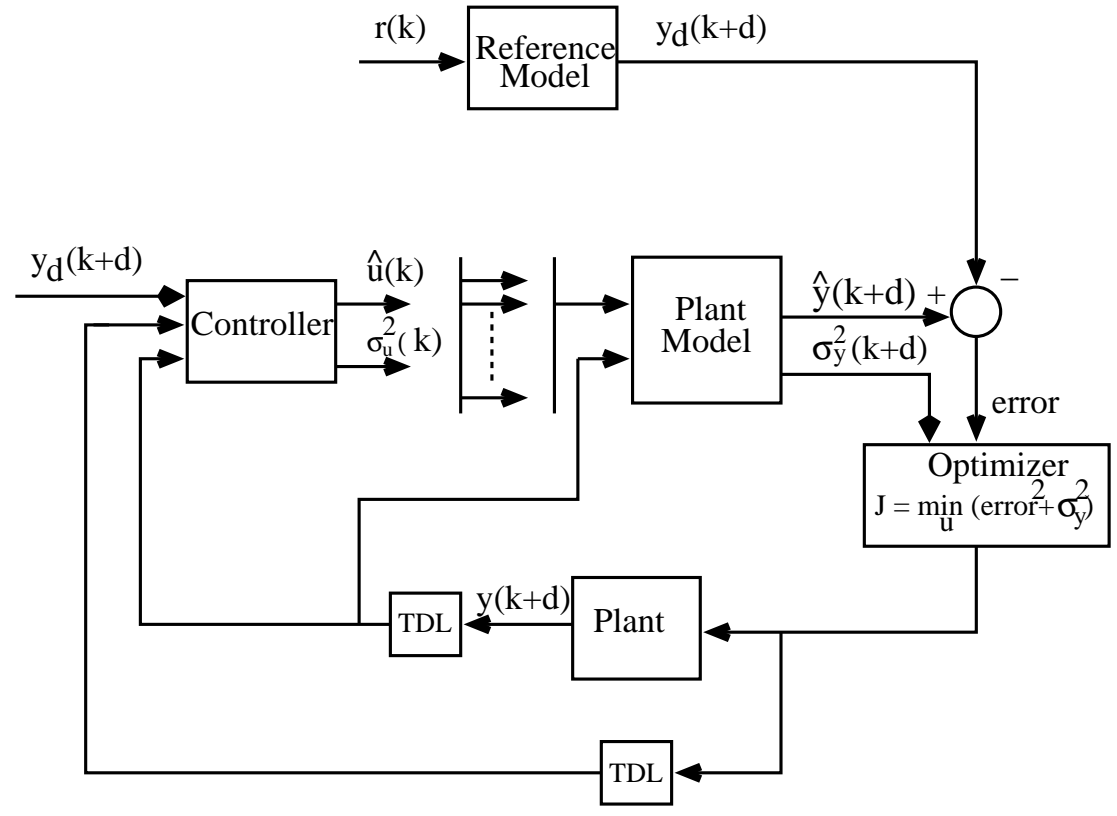

Fig. 3. The architecture of the proposed importance sampling method for the inverse controller.

A likelihood framework for deriving the conditional distribution of the inverse controller has been proposed in [20]. Here, the negative log posterior with respect to the control signal is minimized instead of minimizing the mean squared error function in the conventional direct inverse control method. The proposed scheme was for a general nonlinear plant having the following form

$$
y(k+d)=f(y(k), \ldots, y(k-n), u(k), \ldots, u(k-n-1)),
$$

where $y(k)$ is the measured plant output vector, $u(k)$ is the measured plant input vector, $n$ is the plant order, $d$ is a known plant delay, and $f($.$) is an unknown nonlinear function.$

Based on Theorem 4.2.1 [14] and the result reported in [6], the stochastic forward model of the system given in (2) is firstly identified. Once the forward model of the plant is identified, 
the stochastic forward model can be given by

$$
y(k+d)=\hat{y}(k+d)+e(k+d)
$$

where $e(k+d)$ represents the residual error of the system output which is assumed to be random noise of zero mean and $\sigma^{2}$ variance. This term in other words, represents uncertainty of the forward model. Since the residual error of the system output is assumed to be random with zero mean and $\sigma^{2}$ variance, the distribution of the forward model output could be described by a Gaussian probability density function (pdf) with a global covariance matrix, $R^{-1}$.

$$
p(y(k+d) \mid \mathbf{y}(k), u(k)) \propto \exp \left(-\frac{1}{2}[y(k+d)-\bar{f}(\mathbf{y}(k), u(k))]^{\top} R^{-1}[y(k+d)-\bar{f}(\mathbf{y}(k), u(k))]\right),
$$

where $\mathbf{y}(k)=[y(k), \ldots, y(k-n), u(k-1), \ldots, u(k-n-1)]$ is the vector of previous outputs and inputs values and $\bar{f}(y(k), u(k))$ is the conditional expectation of the forward model.

Given the conditional distribution of the forward model (3), and a Gaussian prior distribution of the control signal denoted by $p\left(u(k) \mid y_{d}(k+d), y(k)\right)$, where $y_{d}(k+d)$ is the desired system output, the posterior probability distribution of the control signal, $p\left(u(k) \mid y(k+d), y_{d}(k+\right.$ $\mathrm{d}), \mathbf{y}(\mathrm{k}))$ is shown, using Bayes rule, to be given by

$$
\begin{aligned}
p\left(u(k) \mid y(k+d), y_{d}(k+d), y(k)\right) & = \\
& \frac{p(y(k+d) \mid u(k), y(k)) p\left(u(k) \mid y_{d}(k+d), \mathbf{y}(k)\right)}{p(y(k+d) \mid \mathbf{y}(k))} .
\end{aligned}
$$

The optimal control law $u^{o p t}(k)$, is then derived by minimizing the negative log posterior of (4) with respect to $u(k)$

$$
\begin{aligned}
& -\log p\left(u(k) \mid y(k+d), y_{d}(k+d), \mathbf{y}(k)\right) \propto \\
& \begin{aligned}
\frac{1}{2}[y(k+d)-\bar{f}(\mathbf{y}(k), u(k))]^{\top} R^{-1}[y(k+d)-\bar{f}(\mathbf{y}(k), u(k))] \\
+\frac{1}{2}[u(k)-\hat{u}(k)]^{\top} \hat{p}^{-1}[u(k)-\hat{u}(k)] \\
\quad-\frac{1}{2}[y(k+d)-\hat{y}(k+d)]^{\top} Q^{-1}[y(k+d)-\hat{y}(k+d)],
\end{aligned}
\end{aligned}
$$

where $\widehat{\mathrm{P}}^{-1}$ is the inverse covariance matrix of the prior distribution of control signals, $\hat{u}(k)$ is the mean of the prior which is equal to the estimated control signal value, $\hat{y}(k+d)$ is the best 
prediction of the system output given an estimate of the control signal, and $\mathrm{Q}^{-1}$ is the inverse covariance matrix of the evidence, $p(y(k+d) \mid \mathbf{y}(k))$.

For linear systems the update equations for the control signal, $\mathrm{u}^{\mathrm{opt}}(\mathrm{k})$, and its variance, $\mathrm{P}^{\mathrm{opt}}$, are shown to be given by [20]

$$
\begin{aligned}
u^{\mathrm{opt}}(\mathrm{k})= & \hat{\mathrm{u}}(\mathrm{k})+\Gamma e(\mathrm{k}), \\
\mathrm{p}^{\mathrm{opt}} & =(\mathrm{I}-\Gamma \widehat{\mathrm{B}}) \widehat{\mathrm{P}},
\end{aligned}
$$

where $\Gamma$ is known as the likelihood gain, $e(k)$ is the error, and $\hat{B}$ is the derivative of the forward linear model with respect to $u(k)$.

For nonlinear systems the optimal control law is shown to be given by taking the derivative of equation (5) with respect to $u(k)$ and setting the derivative equal to zero [20].

$$
[y(k+d)-\bar{f}(y(k), u(k))] \frac{\partial \bar{f}(\mathbf{y}(k), u(k))}{\partial u(k)} R^{-1}=(u(k)-\hat{u}(k)) \hat{P}^{-1}=0 .
$$

A nonlinear optimization method is then used to calculate the optimal control law. The variance of the optimal control signal is shown to be given by,

$$
\mathrm{P}^{\mathrm{opt}}=<\left(\mathrm{u}(\mathrm{k})-\mathrm{u}^{\mathrm{opt}}(\mathrm{k})\right)^{2}>
$$

see [20] for more details.

Although the direct inverse control approach does not require the availability of a forward model of the plant to be controlled, the proposed likelihood method in [20] does. However, the direct inverse control approach in its conventional form does not consider knowledge of uncertainty in deriving the optimal control law, while the likelihood method uses knowledge of uncertainty from both the forward and the inverse models of the plant to obtain the optimal estimate of the control signals. This is an advantage of the likelihood method over the direct inverse control method. Moreover, the likelihood method for deriving the optimal control law provides a systematic procedure for estimating the conditional distribution of the inverse controller. Compared to the proposed sampling approach of the inverse controller [21], one can see that both the likelihood and the sampling approach assume the availability of the forward model of the system to be controlled. However the sampling approach of the inverse controller can be considered to be goal directed, because when generating samples from the inverse controller the error between the forward model output and the desired output is minimized, see equation (1). 
On the other hand, the likelihood approach in its current form could not be considered to be goal directed, although the development of a goal directed likelihood approach is straight forward. The forward model in the likelihood approach is used only to measure effects of the control signal on the system output.

To demonstrates the benefits that could be obtained from incorporating model uncertainty in the direct inverse control architecture we repeat here the example that has been presented in [17], [20], where the plant is taken to be a liquid level system described by the following second order nonlinear equation,

$$
\begin{aligned}
y(k) & =0.9722 y(k-1)+0.3578 u(k-1)-0.1295 u(k-2) \\
& -0.3103 y(k-1) u(k-1)-0.04228 y^{2}(k-2)+0.1663 y(k-2) u(k-2) \\
& -\bar{v} y^{2}(k-1) y(k-2)-0.3513 y^{2}(k-1) u(k-2) \\
& +0.3084 y(k-1) y(k-2) u(k-2)+0.1087 y(k-2) u(k-1) u(k-2),
\end{aligned}
$$

and where $\bar{v}$ is assumed to be a Gaussian random variable $\mathcal{N}(0.03259,0.2)$. The overall performance of the plant under the direct inverse control [26], [50], the proposed sampling approach [21] and the likelihood approach [20] is shown in Figure 4,a,b,c respectively. From this figure it is evident that although the output of the plant increased in an unbounded fashion after running the process for 600 time steps when the direct inverse control is used, the system outputs remain stable in the whole region when the sampling and the likelihood approaches are used.

\section{B. Neural Network Adaptive Control}

The direct inverse control discussed in the previous section in its conventional form lack adaptation and robustness due to the absence of feedback. Recently developed inverse control algorithms which incorporate uncertainty knowledge however, could overcome problems and weaknesses of the inverse controller such that a noticeable improvement on the system performance could be achieved. In the neuro-control field a more robust control scheme than the direct inverse control has been developed. The approach is known as neural adaptive control. It is based upon more accurate and theoretically rigorous considerations than the inverse control class.

In neural adaptive control, a combined off-line followed by on-line adaptation is often adopted to determine the parameter vector of the controller. This reduces prior uncertainty of unknown 


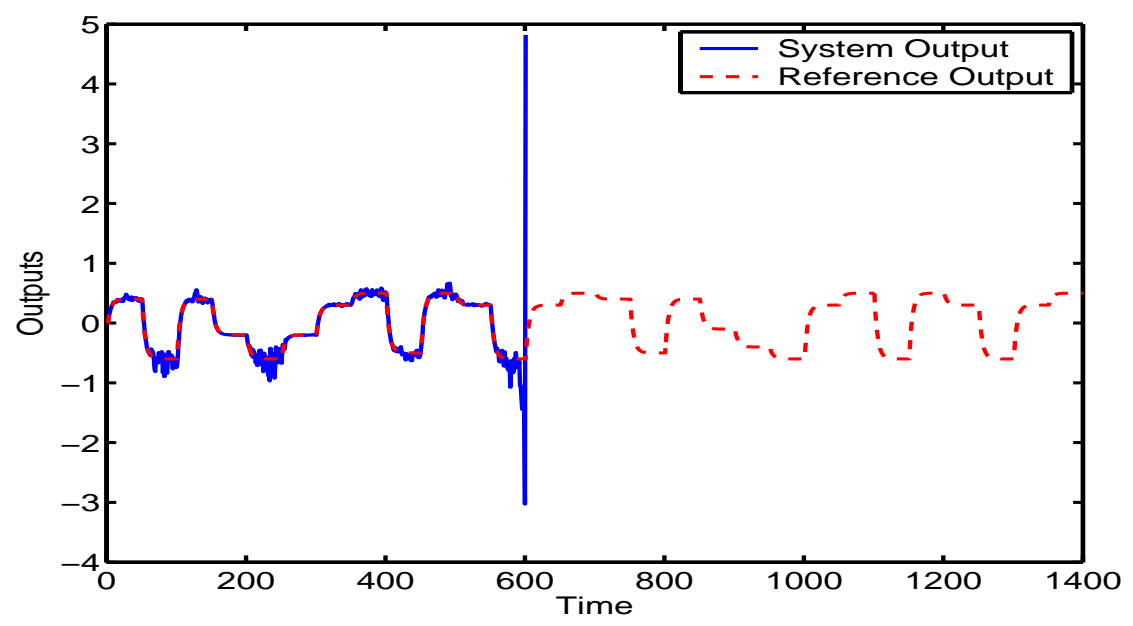

(a)

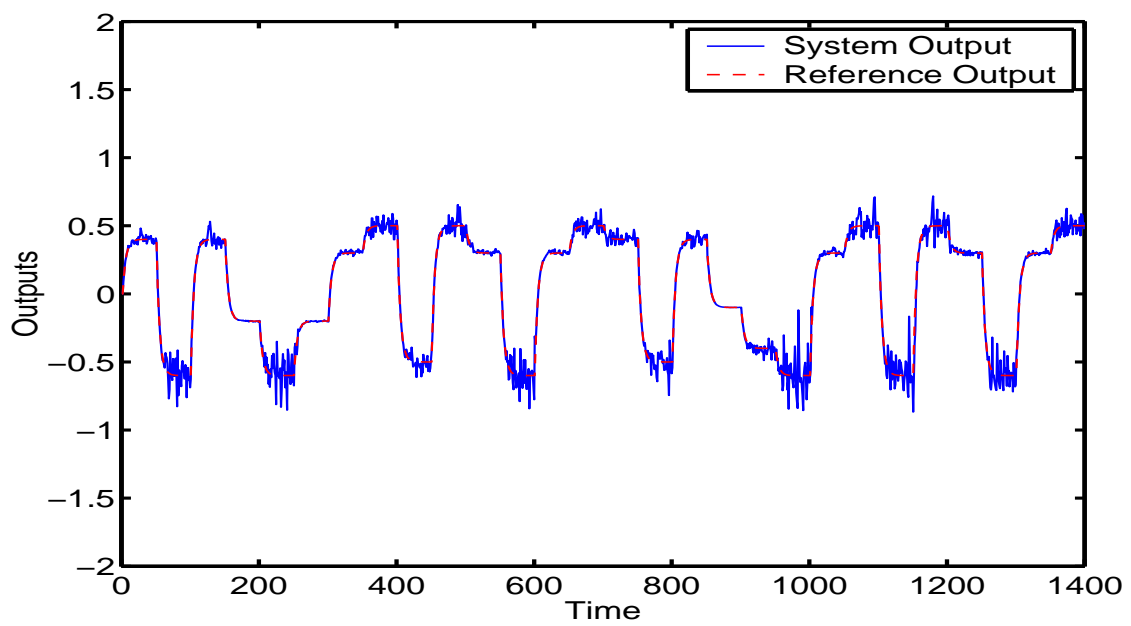

(b)

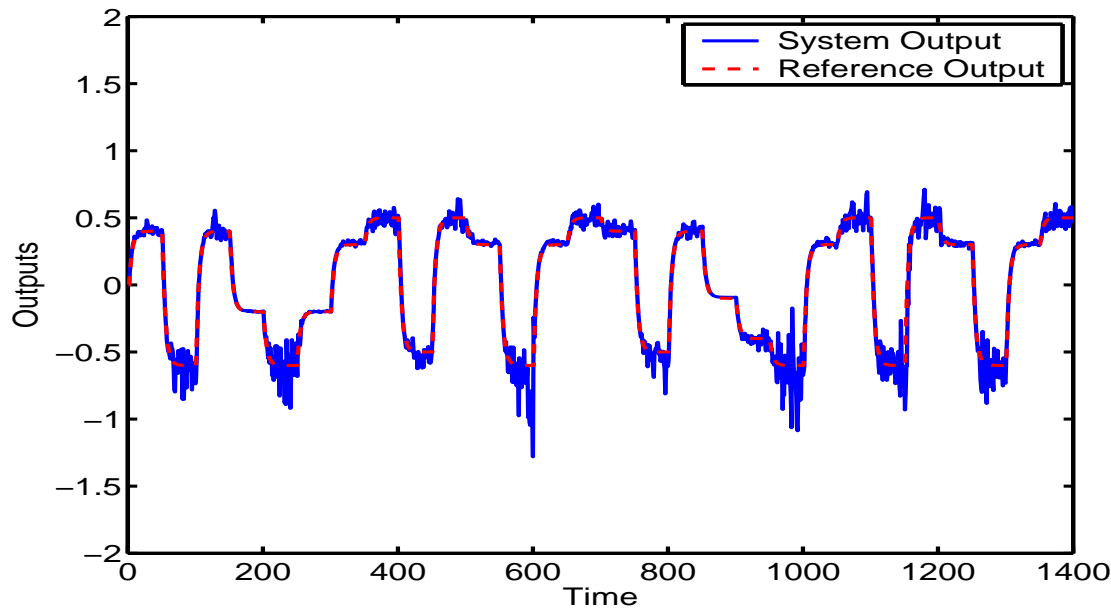

(c)

Fig. 4. Actual and desired outputs for the SISO stochastic process: (a) The desired and actual output values of direct inverse control showing unstable divergence. (b) The desired and actual output values of sampling approach. (c) The desired and actual output values of likelihood approach. Note the use of uncertainty produces stable control. 
parameters and assures stability of the overall control system. Only later on-line control is started using the initial structures of both identifier and controller that are already substantially close to the optimal.

In their book [10], Fabri and Kadirkamanathan made the argument that the above procedure for adaptive control defeats its main objective because most of the uncertainty existing prior to application of the control can be reduced during the off-line training phase. They proposed a dual adaptive control scheme which avoided the pre-control neural network training phase by taking into consideration the parameters' uncertainty and its effect on tracking, in the on-line control phase. Their proposed scheme was for a stochastic affine class of nonlinear discrete time systems having the general form,

$$
y(k)=f[x(k-1)]+g[x(k-1)] u(k-1)+e(k),
$$

where $y(k)$ is the system output, $u(k)$ is the control signal, $x(k-1)=[y(k-n), \ldots, y(k-$ $1), u(k-1-p), \ldots, u(k-2)]^{\top}$ is the vector of previous output and input values, $f[x(k-$ $1)], g[x(k-1)]$ are unknown nonlinear functions of the delay vector and $e(k)$ is an additive noise signal which is assumed to be independent and has zero mean Gaussian distribution of variance $\sigma^{2}$.

Using equation (10) and neural network approximation models for the nonlinear functions of the delay vector, the affine nonlinear discrete system given in (10) is represented in the following state form

$$
\begin{aligned}
w^{*}(k+1) & =w^{*}(k) \\
y(k) & =h\left(w^{*}(k), x(k-1), u(k-1)\right)+e(k),
\end{aligned}
$$

where

$$
h\left(w^{*}, x(k-1), u(k-1)\right)=\hat{f}\left[x(k-1), \hat{w}_{f}\right]+\hat{g}\left[x(k-1), \hat{w}_{g}\right] u(k-1),
$$

could be a nonlinear or linear function of the unknown optimal parameters $w^{*}=\left[w_{f_{1}}^{*}, \ldots, w_{f_{i}}^{*}, w_{g_{1}}^{*}, \ldots, w_{g_{i}}^{*}\right]$ if multi layer perceptron or Gaussian radial basis function networks are used to approximate the nonlinear functions of delay vectors respectively. Since the parameters appear linearly in the Gaussian radial basis controller a Kalman filter is used to estimate the parameters and their uncertainty. The multilayer perceptron controller however, is 
more complicated since the unknown parameters do not appear linearly in the model equations. Consequently, an extended Kalman filter was used for parameter estimation.

Compared to the conventional neural adaptive control methods where the difference between the desired output $y_{d}(k)$, and the system output $y(k),\left[y(k)-y_{d}(k)\right]^{2}$ is minimized to derive the optimal control law as shown in Figure 6,a, the following form of the performance index has been suggested for incorporating model uncertainty,

$$
J=E\left\{\left[y(k)-y_{d}(k)\right]^{2}+q u^{2}(k-1)+r e^{2}(k) \mid I^{k-1}\right\},
$$

where $E\left\{. \mid I^{k-1}\right\}$ denotes the mathematical expectation conditioned on the information state $I^{k-1}$ which consists of all output measurements up to time $(\mathrm{k}-1)$, denoted by $\mathrm{Y}^{\mathrm{k}-1}=\{\mathrm{y}(\mathfrak{i})\}_{\mathfrak{i}=0}^{\mathrm{k}-1}$, and all previous inputs $\mathrm{U}^{\mathrm{k}-2}$. The design parameters $r$, and $\mathrm{q}$ are scalar weighting factors.

The control law minimizing the above performance index J subject to the system equation (10), is then shown to be given by

$$
u^{*}(k-1)=\frac{\left\{y_{d}(k)-\hat{f}[.]\right\} \hat{g}[.]-(1+r) \mu_{g f}}{\hat{g}^{2}[.]+q+(1+r) \mu_{g g}},
$$

where the arguments [.] of $\hat{f}$ and $\hat{g}$ are $\left[x(k-1), \hat{w}_{f}(k)\right]$ and $\left[x(k-1), \hat{w}_{g}(k)\right]$ respectively,

$$
\begin{aligned}
& \mu_{g f}=\nabla_{h_{g}}(k), P_{g f}(k) \nabla_{h_{f}}^{\top}(k) \\
& \mu_{g g}=\nabla_{h_{g}}(k) P_{g g}(k) \nabla_{h_{g}}^{\top}(k),
\end{aligned}
$$

where $P_{g f}$, and $P_{g g}$ are the partitioning matrices of the covariance matrix of the optimal neural network parameters $w^{*}$ and $\nabla_{h_{g}}, \nabla_{h_{f}}$ denote the gradients of the two components of the function $h, \hat{f}$ and $\hat{g}$, with respect to $w^{*}$ evaluated at $w^{*}=\hat{w}(k)$ respectively [10].

In the above proposed scheme [10] Fabri and Kadirkamanathan avoided the pre-control neural network phase by taking into consideration model parameter uncertainty. This is shown to be more convenient with the features expected from the adaptive control, and also more efficient and economical since off-line training is usually time consuming and expensive.

An alternative approach for incorporating uncertainty in functional adaptive control by neural networks was proposed in [17]. The scheme is based on the idea of modeling and incorporating the uncertainty in the predicted output of the neural network model. Here the forward model of the plant is firstly identified using a neural network model. Similar to the discussion in 
Section III-A and based on theorem 4.2.1 [14], the output of the system is shown to be given by [17]

$$
y(k)=\hat{y}(k)+e(k),
$$

where $\hat{y}(k)$ is the conditional expectation of the system output modeled using a neural network or any function approximator, and $e(k)$ is the residual error of the output which is shown to be a random variable with zero mean Gaussian distribution of variance equal to the squared difference between the system output and its estimate, $\|\mathrm{y}-\hat{\mathrm{y}}\|^{2}$. This variance is input dependent as has been discussed in Section III-A. The conditional expectation of this variance is modeled using another neural network [17].

Since the estimated variance, $\sigma^{2}$, around the predicted output of the system model is input dependent, Herzallah [17] has shown that the derived control law from the conventional neural adaptive control method is not optimal. Instead of minimizing the difference between the system and the desired outputs, $\left\|y(k)-y_{d}(k)\right\|^{2}$ in the conventional adaptive control, it has been shown [17] that a performance index of the following form should be minimized,

$$
\begin{aligned}
& J=E\left\{\left(y(k)-y_{d}(k)\right)^{2}\right\}, \\
& =\left(y(k)-y_{d}(k)\right)^{2}+\sigma^{2} .
\end{aligned}
$$

Hence, dropping off the variance of the system output which is also input dependent from the performance index to be minimized in deriving the control law can in no way give the optimal solution. Consequently the optimal control law is shown to be given by differentiation of (17) with respect to $u(k)$ and equating to zero.

$$
\frac{\partial J}{\partial u(k)}=\left(y(k)-y_{d}(k)\right) \frac{\partial y(k)}{\partial u(k)}+\frac{\partial \sigma^{2}}{\partial u(k)}=0 .
$$

This Control law and the control law proposed by Fabri and Kadirkamanathan [10] take into consideration uncertainty of the forward model only, and ignore uncertainty of the inverse model.

An alternative control algorithm which takes uncertainty of the forward and inverse models into consideration is proposed in [19]. The architecture of this proposed method is shown in Figure 6,b. It is of an explicit type, sub-optimal dual performance index based on the innovations dual controller developed by Fabri and Kadirkamanathan [10] for a class of stochastic singleinput single-output affine nonlinear systems. The performance index in [19] is modified such 
that uncertainty in the inverse controller model is taken into consideration as well as uncertainty in the forward model output. Hence the performance index is taken to have the form

$$
J=E\left\{\left[y(k+d)-y_{d}(k+d)\right]^{2}+r e_{y(k+d)}^{2}+\tau u^{2}(k)+\lambda e_{u(k)}^{2} \mid I^{k}\right\},
$$

where $E\left\{. \mid I^{k}\right\}$ denotes mathematical expectation conditioned on the information state $I^{k}$ which consists of all output measurements up to time $(k)$, denoted by $Y^{k}=\{y(i)\}_{i=0}^{k}$, and all previous inputs $\mathrm{U}^{k-1}$. The design parameters $r, \tau$, and $\lambda$ are scalar weighting factors chosen within the range $\tau \geq 0,-1 \leq r \leq 0,0 \leq \lambda \leq 1$.

The difference between this performance index and that originally proposed in [10], is the inclusion of the term $\lambda e_{\mathfrak{u}(\mathrm{k})}^{2}$. This term reflects our knowledge about uncertainty of the inverse controller.

The optimal control law is then derived by minimization of the sub-optimal dual performance index given in Equation (19). Taking the assumptions of Gaussian distributions of the residual errors of the forward and inverse models, and using the general result that for a Gaussian random variable $\eta, E\left\{\eta^{2}\right\}=[E\{\eta\}]^{2}+$ variance $\{\eta\}$, the sub-optimal performance dual index given in Equation (19) can be rewritten as

$$
J=\left[\hat{y}(k+d)-y_{d}(k+d)\right]^{2}+(1+r) \sigma_{y(k+d)}^{2}+\tau u^{2}(k)+\lambda \sigma_{u(k)}^{2} \text {. }
$$

Taking the derivative of this equation with respect to $u(k)$ and setting the derivative equal to zero yields,

$$
\begin{aligned}
2\left[\hat{y}(k+d)-y_{d}(k+d)\right] \frac{\partial \hat{y}(k+d)}{\partial u(k)}+(1+r) \frac{\partial \sigma_{y(k+d)}^{2}}{\partial u(k)} & \\
& +2 \tau u(k)+\lambda \frac{\partial \sigma_{u(k)}^{2}}{\partial \hat{y}(k+d)} \frac{\partial \hat{y}(k+d)}{\partial u(k)}=0 .
\end{aligned}
$$

This equation is then solved for the optimal control law. The parameters of the inverse controller are then adapted such as to predict the conditional expectation of this optimal control law. The parameters of the network which model the variance of the inverse controller have also to be adapted to minimize the squared difference between the optimal control law and its estimate.

We repeat here the example in [19] to demonstrate the advantages of incorporating model uncertainty in the functional adaptive control scheme. The dynamic equation of the system was 
taken to be described by

$$
y(k+1)=\sin [y(k)]+\cos [3 y(k)]+\{2+\cos [y(k)]\} u(k)+e(k+1) .
$$

where $e(k+1)$ was assumed to be sampled from a Gaussian distribution, $\mathcal{N}(0,0.001)$. In [19], three experiments were conducted using three different design parameter settings corresponding to certainty equivalence $(r=-1, \lambda=0)$, cautious $(r=0, \lambda=1)$ and dual control $(r=$ $-0.6, \lambda=0.8$ ). In all experiments on-line control was started immediately and the neural networks were never subjected to initial off-line training phase. The three experiments were simulated using the same initial conditions, noise sequence, reference input, and control penalty $\tau=0.0001$. The plant and desired outputs resulting from each experiment are shown in Figure 5 . As can be seen from this figure and following the discussion in [19] the large transient error results from the certainty equivalence controller is expected because model uncertainties are not considered in calculating the control signal of this controller. The cautious controller on the other hand, does not overreact during the transient period knowing that model parameter estimates are still inaccurate. However as can be seen from the figure, the cautious controller is almost inactive during the first period of control and takes longer time to track the desired output. The best results could be obtained from the dual controller. It strikes a compromise between the slow tracking performance of the cautious controller and at the same time shows no large overshoots in the transient period [19].

Compared to the method proposed by [10] this method can be seen to be more general for many reasons. Firstly, this method is shown to be suitable for the four different classes of models defined by Narendra and Parthasarathy in [48], as long as the variance of the forward model could be estimated as an input dependent variance. The method proposed in [10] on the other hand was for a specific affine class of nonlinear discrete time systems. Secondly, the variance of the residual error in [19] is the variance of the error of the predicted output from the neural network. This includes all possible sources of variation in the predicted output, whether it is due to noise affecting the output, noise affecting the input, or even due to parameter uncertainty. The variance in [10] however, includes variations of model parameters only. 


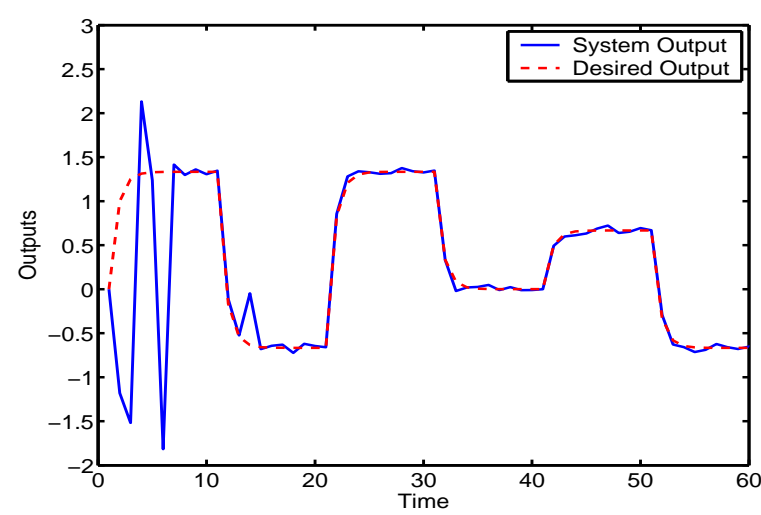

(a)

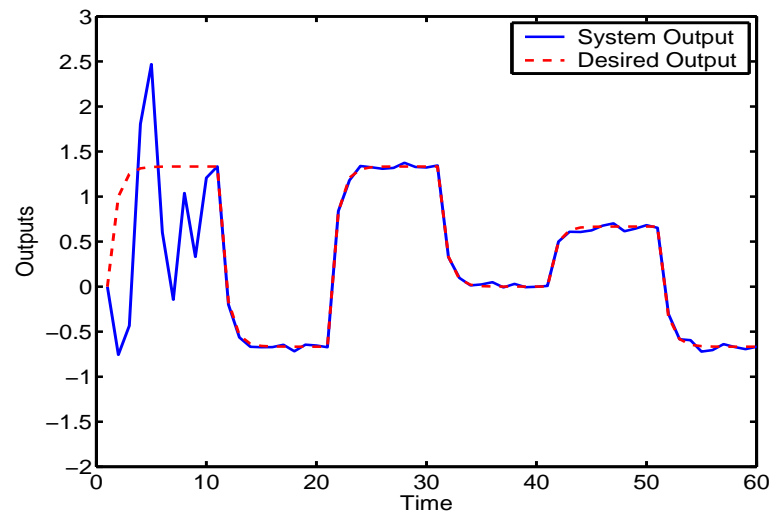

(c)

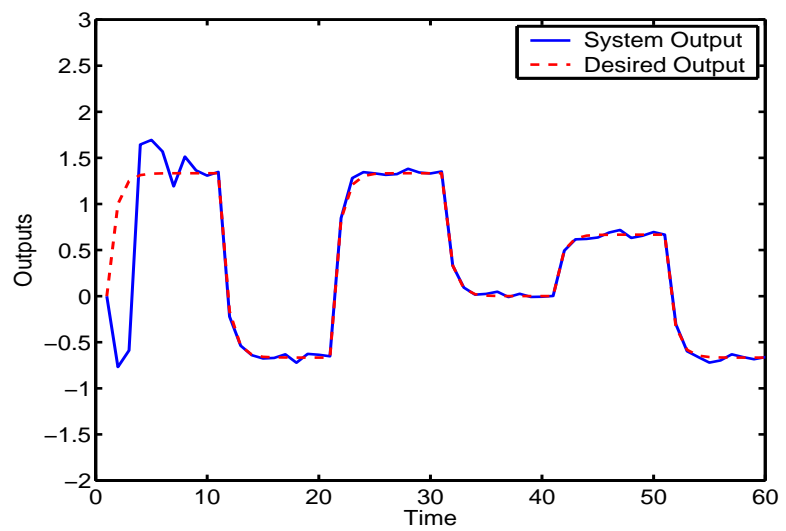

(e)

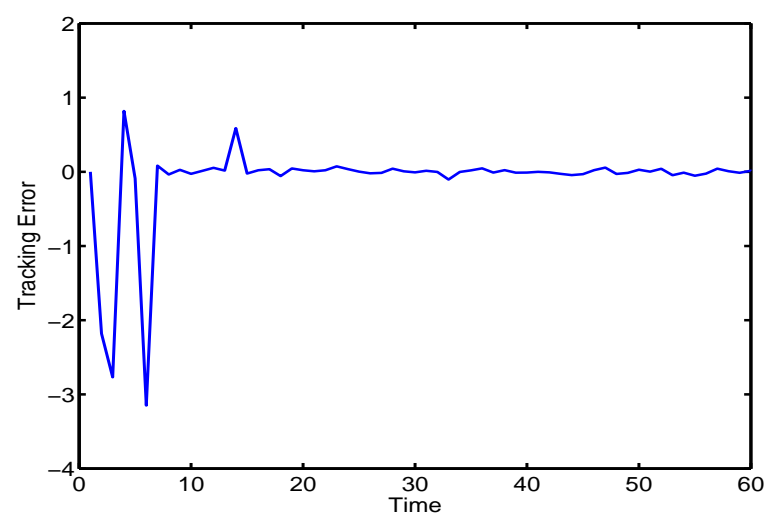

(b)

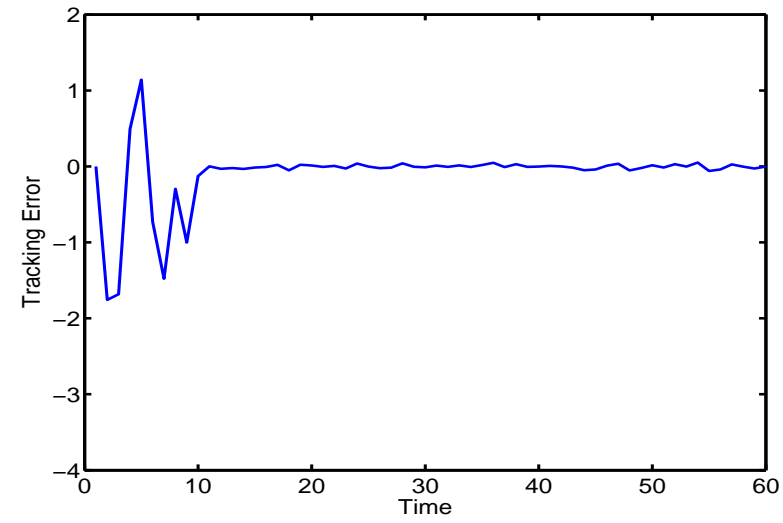

(d)

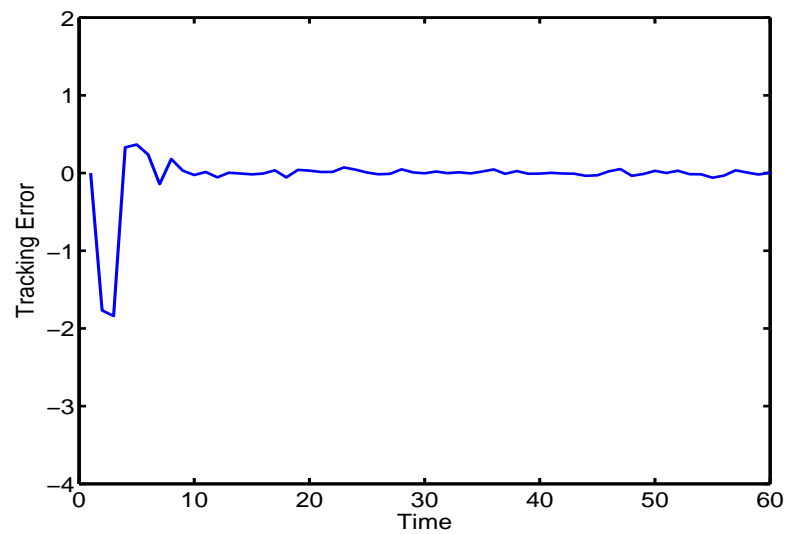

(f)

Fig. 5. Control Results of the single-input single-output nonlinear stochastic control system using three different control methods: (a) The actual and desired model outputs using certain equivalence control method. (b) The tracking error from the certain equivalence method. (c) The actual and desired model outputs using the cautious control method. (d) The tracking error from the cautious method. (e) The actual and desired model outputs using the dual control method. (f) The tracking error from the dual method. 


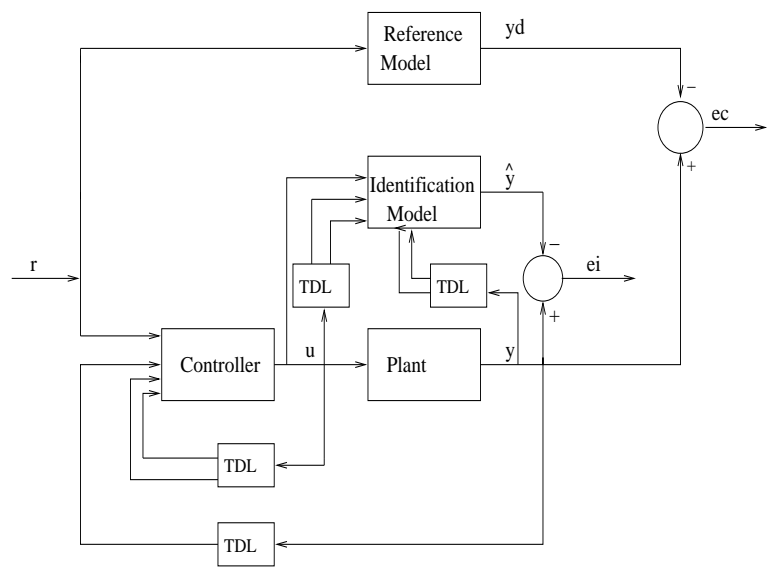

(a)

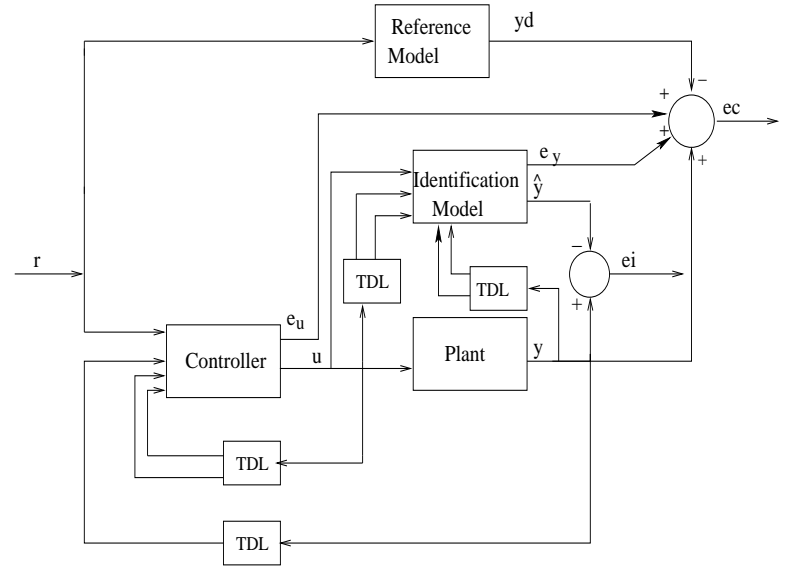

(b)

Fig. 6. The architectures of conventional indirect adaptive control and indirect adaptive control which incorporates model uncertainty: (a) Conventional indirect adaptive control using a neural network, here the error between the identification model and the desired output is used to adapt the inverse controller. (b) Indirect adaptive control which incorporates model uncertainty, as can be seen uncertainty of the forward and inverse models in addition to the error between the identification model and the desired output are used to adapt the inverse controller.

\section{Adaptive Critic Control}

The neuro-control community has developed a general family of control designs capable of planning or optimization over time in complex way and has the ability to cope with noise. So unlike the neural adaptive and the inverse control algorithms, adaptive critic neural networks show real promise of deriving the optimal control law for complex processes under uncertain conditions.

This approach can be defined as a set of methods that approximate dynamic programming. The method is based on the basic concept common to all forms of dynamic programming [24]. The user needs to supply a utility function $\mathrm{U}$ and a stochastic model of the plant to be controlled. Dynamic programming is used to solve for another function called the cost function J, which is assumed to be a function of the state variable at time $k$ of the plant to be controlled, $x(k)$.

Following the concept of dynamic programming, adaptive critic methods can be defined more precisely as designs that include two neural networks: the critic network which tries to approximate the cost function J or its derivatives, and the action network which should be adapted so as to maximize $J$ in the near term future. As shown in Figure 7, the input to both the action and the critic networks is the state vector $x(k)$. The cost function to be minimized is usually 
taken to be of the following form

$$
\mathrm{J}[x(k)]=\mathrm{U}(x(k), u[x(k)])+<J[x(k+1)]>.
$$

Based on the output supposed to be approximated by the critic network and the method for adapting the action network, three different critic designs have been proposed in the literature: (1) Heuristic dynamic programming (HDP), which adapts a critic network whose output is an approximation of $\mathrm{J}(\mathrm{x}(\mathrm{k}))$, (2) Dual heuristic programming (DHP), which adapts a critic network whose outputs represent the derivative of $J(x(k))$ [3], and (3) Globalized DHP (GDHP), which adapts a critic network whose output is an approximation of $\mathrm{J}(\mathrm{x}(\mathrm{k}))$, but adapts it so as to minimise errors in the implied derivatives of J, as well as J itself. The reader is referred to [49], [56] for full discussion about critic designs.

The adaptive critic design methods are capable of deriving near-optimal control laws over time in noisy nonlinear environments and under uncertain conditions. This comes from the fact that the adaptive critic methods are an approximation for dynamic programming which is currently the only mathematical formalism under which an optimal controller can be designed under uncertain conditions. The fact that (23) takes the expected value of the cost function at time $k+1,<J[x(k+1)]>$, shows that model uncertainty can be accounted for in deriving the near-optimal control law, although non of the new research considers this.

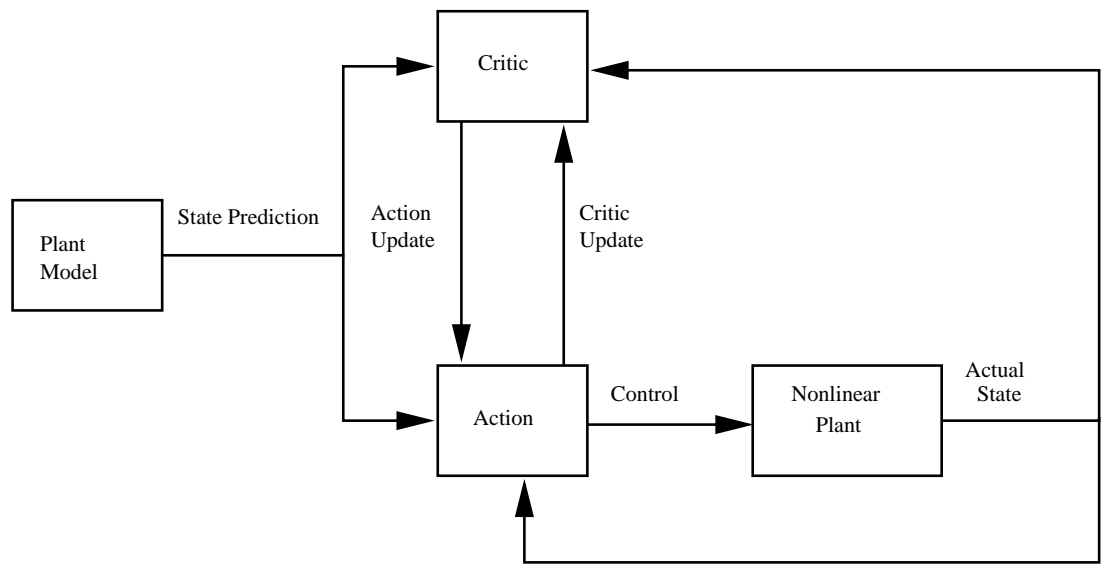

Fig. 7. An adaptive critic design. The outputs of the critic network are the derivative of $J(x(k))$

Recently Herzallah [18] has proposed a new control algorithm for the adaptive critic networks which takes into consideration model uncertainty. Critic methods usually assume the availability 
of a forward model when calculating the control law. The argument raised in [18] was that, although an approximation model is usually identified so as to represent the forward model of the plant, researchers in the adaptive critic field usually assume that the obtained forward model is accurate and ignore uncertainty of that model. Consequently, a cautious type adaptive critic controller which takes uncertainty of a models' estimate into consideration when calculating the control law was proposed. The proposed controller has been obtained directly by ideally solving the adaptive critic problem. In contrast to how the adaptive critic problem is usually used so as to derive the control law, certainty equivalence is not assumed in the cautious adaptive critic controller [18]. The control law is derived by minimization of the performance index $J[x(k)]$ given in (23), but with the uncertainty of the model estimates taken into consideration by treating the predicted state vector or output of the system as random variables. Taking the assumptions of Gaussian distributions of the forward model, the fact that the cost function $\mathrm{J}[\mathrm{x}(\mathrm{k})]$ is a quadratic function in $x(k)$, and using the general result that for a random variable $\eta, E\left\{\eta^{2}\right\}=[E\{\eta\}]^{2}+$ variance $\{\eta\}$, the sub-optimal performance index given in Equation (23) is shown to be given by [18]

$$
\begin{aligned}
\mathrm{J}[\mathrm{x}(\mathrm{k})] & =\mathrm{U}(\mathrm{x}(\mathrm{k}), \mathrm{u}[\mathrm{x}(\mathrm{k})]) \\
& +<\mathrm{J}[\hat{x}(\mathrm{k}+1)+e(k+1)]> \\
& =\mathrm{U}(x(k), \mathrm{u}[x(k)])+\mathrm{J}[\hat{x}(k+1)]+\operatorname{tr} \Sigma M .
\end{aligned}
$$

where tr denotes the trace of the matrix, $M$ is the weighting matrix associated with the cost function $\mathrm{J}[x(k)]$ which can be calculated if required, and $\Sigma$ is the covariance matrix of the residual error.

Since $\Sigma$ is state dependent, the derivative of the above cost function with respect to the state $x(k)$ at time $k$, defined as $\lambda[x(k)]$ is shown to be given by,

$$
\begin{aligned}
\lambda[x(k)] \equiv \frac{\delta J[x(k)]}{\delta x(k)}=\frac{\partial u[x(k), u(k)]}{\partial x(k)} & +\frac{\partial u[x(k), u(k)]}{\partial u(k)} \frac{\partial u[x(k)]}{\partial x(k)} \\
+\lambda[x(k+1)] \frac{\partial x(k+1)}{\partial x(k)} & \\
& +\lambda[x(k+1)] \frac{\partial x(k+1)}{\partial u(k)} \frac{\partial u[x(k)]}{\partial x(k)}+\operatorname{tr} \frac{\partial \Sigma}{\partial x(k)} M .
\end{aligned}
$$

The covariance of the forward model $\Sigma$ is dependent on the control signal as well, so the 
optimality equation is shown to be given by,

$$
\begin{aligned}
\frac{\partial J[x(k)]}{\partial u(k)} & =\frac{\partial u[x(k), u(k)]}{\partial u(k)} \\
& +\lambda[x(k+1)] \frac{\partial x(k+1)}{\partial u(k)}+\operatorname{tr} \frac{\partial \Sigma}{\partial u(k)} M \\
& =0 .
\end{aligned}
$$

The training process for the cautious type adaptive critic proposed in [18] is exactly the same as that described for the adaptive critic in its conventional form. The only difference is that (25) is used here to calculate the target of the critic network, and the output from the converged critic is used in (26) solving for the target $\mathrm{u}^{*}(\mathrm{k})$ which is then used to correct the action network. In addition the proposed method is recommended to be implemented on-line. The forward model of the plant to be controlled, the controller and the critic networks can all be adapted on-line. This is because the proposed adaptive-critic-based cautious controller considers model uncertainty in calculating the control law.

Compared to the conventional adaptive critic networks, the cautious adaptive critic network proposed by Herzallah [18] has the advantage of incorporating model uncertainty when deriving the optimal control law.

In the same way, the example that has been presented in [18] is repeated here. The plant equation was assumed to be a linear input-output stochastic model given by,

$$
x(k+1)=x(k)+2 u(k)+\text { noise },
$$

where the noise term is assumed to be sampled from a Gaussian distribution, $\mathcal{N}(0,0.02)$. Following the procedure in [18] Figure 8 shows a comparison of the system state being controlled by both the optimal control using the policy method of dynamic programming which accounts for model uncertainty in calculating the control law by considering state and control signal dependent variance and the control determined by the proposed cautious adaptive critic method for $x(0)=-20$. The results in this figure are obtained by performing on-line identification for the proposed adaptive critic method only. For the policy method of dynamic programming, off-line identification for calculating the optimal control law is assumed [18]. From this figure it is clear that good control results could be obtained from the cautious adaptive critic method which incorporates model uncertainty. 


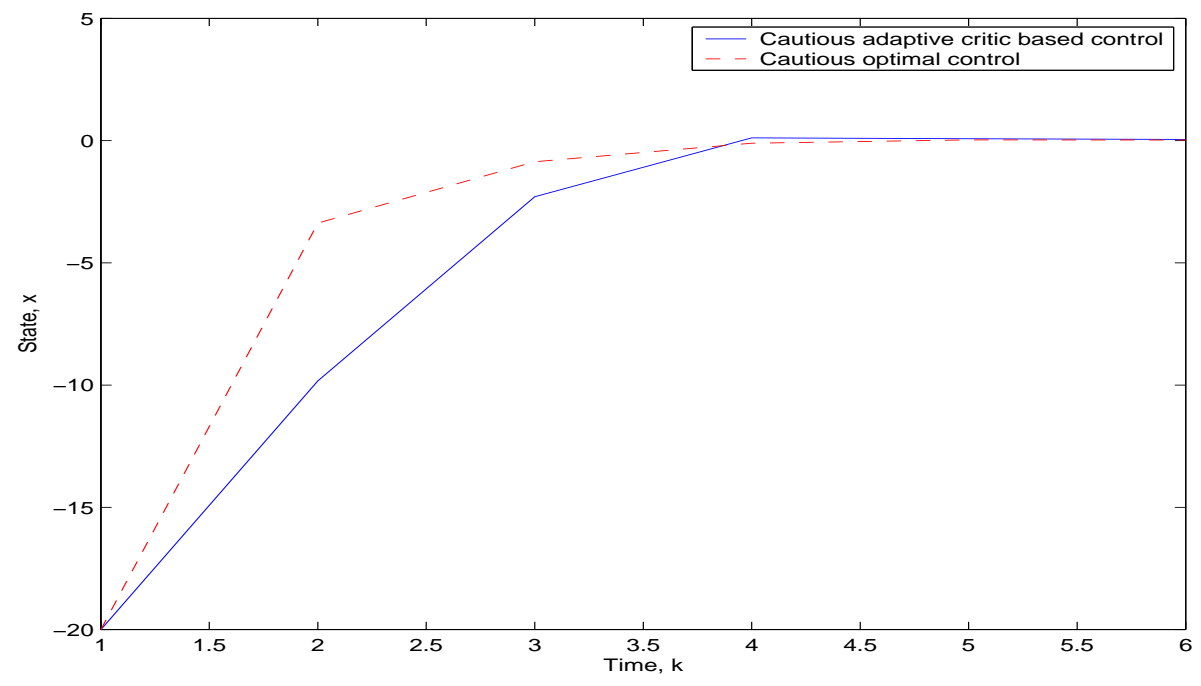

Fig. 8. Controlled linear input output stochastic system with initial condition $x(0)=-20$. It can be seen that that the cautious adaptive critic method produces stable control results and it could bring the state to zero as required, even though the forward model parameters, the parameters of the inverse controller and the critic network parameters are all adapted on-line without any prior off-line training.

IV. Improving the Performance of Intelligent Control Using MUltiple models: DEALING WITH UNCERTAINTY

As discussed in Section II, multiple model approaches have been proposed to handle problems with higher levels of uncertainty and complexity, known as multi-modality.

In the control literature, three types of multi-modality are considered. The first one is temporal multi-modality: this situation occurs when the plant operates in suddenly changing environments or when a fault condition occurs. The second type of multi-modality is called spatial multimodality: it occurs when the plant is characterized by a highly nonlinear complex function, which exhibits different characteristics over different operating zones or operating spaces. The third type of multi-modality occurs when one tries to acquire the inverse dynamics of the plant using supervised learning. This is an ill posed problem, where there is a well defined forward solution, but the solutions to the inverse problem are not unique. Most motor control problems are ill-posed in the sense that there is a well defined forward solution, but the inverse solution is not unique.

Although multiple approaches usually model the conditional probability of the $i^{\text {th }}$ model, 
the controller is usually designed by ignoring knowledge of uncertainty. Two different methods have been suggested for designing the controller. In the first method the new control signal is taken to be the output of the controller with the highest conditional probability. In the second method, the new control signal is taken to be the probability weighted average of the outputs of all controllers.

Different methods for incorporating uncertainty knowledge in the multiple model approaches have recently appeared in the control literature. In the following we discuss briefly two of the approaches.

The first method is a mixture of adaptive control to handle dynamic uncertainty, and multiple model techniques to handle the multi-modality. This method is known as a multiple model adaptive control scheme [10]. It is designed for a class of affine-nonlinear stochastic plant with temporal multi-modality of the following form

$$
y(k)=f_{m(k)}[x(k-1)]+g_{m(k)}[x(k-1)] u(k-1)+e(k),
$$

where $y(k)$ is the system output, $u(k)$ is the control signal, $x(k-1)=[y(k-n), \ldots, y(k-$ $1), u(k-1-p), \ldots, u(k-2)]^{\top}$ is the vector of previous output and input values, and $e(k)$ is an additive noise signal which is assumed to be independent and has zero mean Gaussian distribution of variance $\sigma^{2}$. The smooth nonlinear functions $f_{m(k)}[x(k-1)], g_{m(k)}[x(k-1)]$ could switch form at an arbitrary instant in time taking on any of the pairs $\left\{\left(f_{1}, g_{1}\right),\left(f_{2}, g_{2}\right), \ldots,\left(f_{H}, g_{H}\right)\right\}$ as indexed by $m(k) \in\{1, \ldots, H\}$.

A multiple model approach based on a Gaussian radial basis function network is used to identify the nonlinear modes of the plant. $\mathrm{H}$ local neural network models, one per mode, are then used to identify the plant and to control it via indirect adaptive techniques. Two Gaussian radial basis function networks are used for each local model to identify the two nonlinear functions $\left(f_{i}\right)$ and $\left(g_{i}\right)$ in $(28)$,

$$
\begin{aligned}
\hat{f}_{i}\left[x, \hat{w}_{f_{i}}^{\top}\right] & =\hat{w}_{f_{i}}^{\top} \phi_{f_{i}}[x], \\
\hat{g}_{i}\left[x, \hat{w}_{g_{i}}^{\top}\right] & =\hat{w}_{g_{i}}^{\top} \phi_{g_{i}}[x] .
\end{aligned}
$$

As can be seen from the above equation, the unknown variables consist of the optimal output layer parameters of the networks in all local models, $w_{f_{i}}^{*}, w_{g_{i}}^{*} ; i=1, \ldots, H$. 
From (28) and (29), the system dynamics during activity of the mode captured by local model $i$ could be represented in the following state space form

$$
\begin{aligned}
w_{i}^{*}(k+1) & =w_{i}^{*}(k), \\
y(k) & =w_{i}^{*^{\top}}(k) \phi_{i}[x(k-1)]+e(k),
\end{aligned}
$$

where $w_{i}^{*^{\top}}=\left[w_{f_{i}}^{*^{\top}}(k), w_{g_{i}}^{*^{\top}}(k)\right]$ and $\phi_{i}^{\top}[x(k-1)]=\left[\phi_{f_{i}}^{\top}[x(k-1)] \phi_{g_{i}}^{\top}[x(k-1)] u(k-1)\right]$.

The number of local models to be estimated is determined by the number of plant modes if known a priori. Otherwise, a self organized scheme which allows adding new local models is used [10]. Since (30) is linear in the parameters, a Kalman filter is used to generate recursively the conditional minimum mean square predictive estimate $\hat{w}_{i}(k+1)$ of $w_{i}^{*}$ and its covariance matrix $P_{i}(k+1)$ whenever the mode corresponding to local model $i$ is active as could be detected by $m(k)$. As the mode index $m(k)$ is not actually known a mode estimation method is developed in [10]. Interested readers are referred to [10] for the problem of mode and parameter estimation.

Following the discussion in Section III-B a performance index of the form given in (14) has been suggested for incorporating model uncertainty in the multiple model adaptive control scheme [10]. The difference here is that a number of local models are taken to represent the forward dynamics of the system as can be seen from (28).

Subject to (28) and knowledge of the mode sequence $S(k)=\{m(1), m(2), \ldots, m(k)\}$, the control law minimizing the performance index J stated in (14) is then shown to be given by

$$
u^{*}(k-1)=\frac{\left\{y_{d}(k)-\hat{f}_{m(k)}[.]\right\} \hat{g}_{m(k)}[.]-(1+r) v_{g f_{m(k)}}}{\hat{g}_{m(k)}^{2}[.]+q+(1+r) v_{g g_{m}(k)}},
$$

where

$$
\begin{aligned}
\hat{f}_{m}(k) & =\hat{w}_{f_{m(k)}}^{\top}(k \mid S(k)) \phi_{f}[x(k)], \\
\hat{g}_{m}(k) & =\hat{w}_{g_{m}(k)}^{\top}(k \mid S(k)) \phi_{g}[x(k)], \\
v_{g f_{m(k)}} & =\phi_{g}^{\top}[x(k)] P_{g f_{m(k)}}(k \mid S(k)) \phi_{f}[x(k)], \\
v_{g g_{m(k)}} & =\phi_{g}^{\top}[x(k)] P_{g_{g_{m}(k)}}(k \mid S(k)) \phi_{g}[x(k)],
\end{aligned}
$$

and where $\hat{w}_{f_{m(k)}}(k \mid S(k))$ and $\hat{w}_{g_{m}(k)}(k \mid S(k))$ are sub-vectors of $\hat{w}_{m(k)}(k \mid S(k))$. Similarly $P_{g f_{m(k)}}(k \mid S(k))$ and $P_{g g_{m(k)}}(k \mid S(k))$ are sub-matrices of the covariance matrix $P_{m(k)}(k \mid S(k))$. 
Compared to the conventional multiple model approaches, this approach has the advantage of incorporating uncertainty of model parameters. Again only parameter uncertainty is accounted for in this approach. All other sources of uncertainty have been ignored.

The second method uses the mixture density network approach for representing general probability density functions of the inverse controllers. This method is applied to ill-posed control problems in which the solution to the inverse controller is not unique [21].

For multi-valued functions (ill-posed problems), it has been shown [6], [21] that mixture density networks (MDNs) provide a general framework for modeling the conditional probability density functions of inverse controllers, $p(u(k) \mid s(k))$. Here $s(k)=\left[y_{d}(k+d), x(k)\right]$, where $x(k)=[y(k), \ldots, y(k-q+1), u(k-1), \ldots, u(k-p+1)]$. The distribution of the control signals, $u(k)$, is described by a parametric model whose parameters are determined by the output of a neural network, which takes $s(k)$ as inputs. The general conditional distribution function is given by

$$
p(u(k) \mid s(k))=\sum_{j=1}^{M} \alpha_{j}(s(k)) \phi(u(k) \mid s(k)),
$$

where $\alpha_{j}(s(k))$ represents the mixing coefficients, and can be regarded as prior probabilities (which depend on $s(k)$ ), $\phi_{j}(u(k) \mid s(k))$ are the kernel distributions of the mixture model (whose parameters are also conditioned on $s(k))$, and $M$ is the number of kernels in the mixture model. This combination of a density model and a feed-forward neural network is represented schematically in Fig 9.

Different methods for using the output from the mixture density network have been suggested. For control applications where unique solutions cannot be found, and where the distribution of the target data consists of different numbers of distinct branches, one specific branch from the estimated conditional density of the MDN needs to be selected. Two examples of how to select a specific branch are the most likely, and the most probable output values. Interested readers are referred to [6], [17] for more details.

However it has been shown [21] that neither of the two proposed methods lead to the optimal control law. The argument was based on the fact that although mixture density networks model the general distribution of the inverse controllers, specific quantities are then selected to represent the output of the mixture density network, either the most probable or the most likely value, and all other information about uncertainty is ignored. 

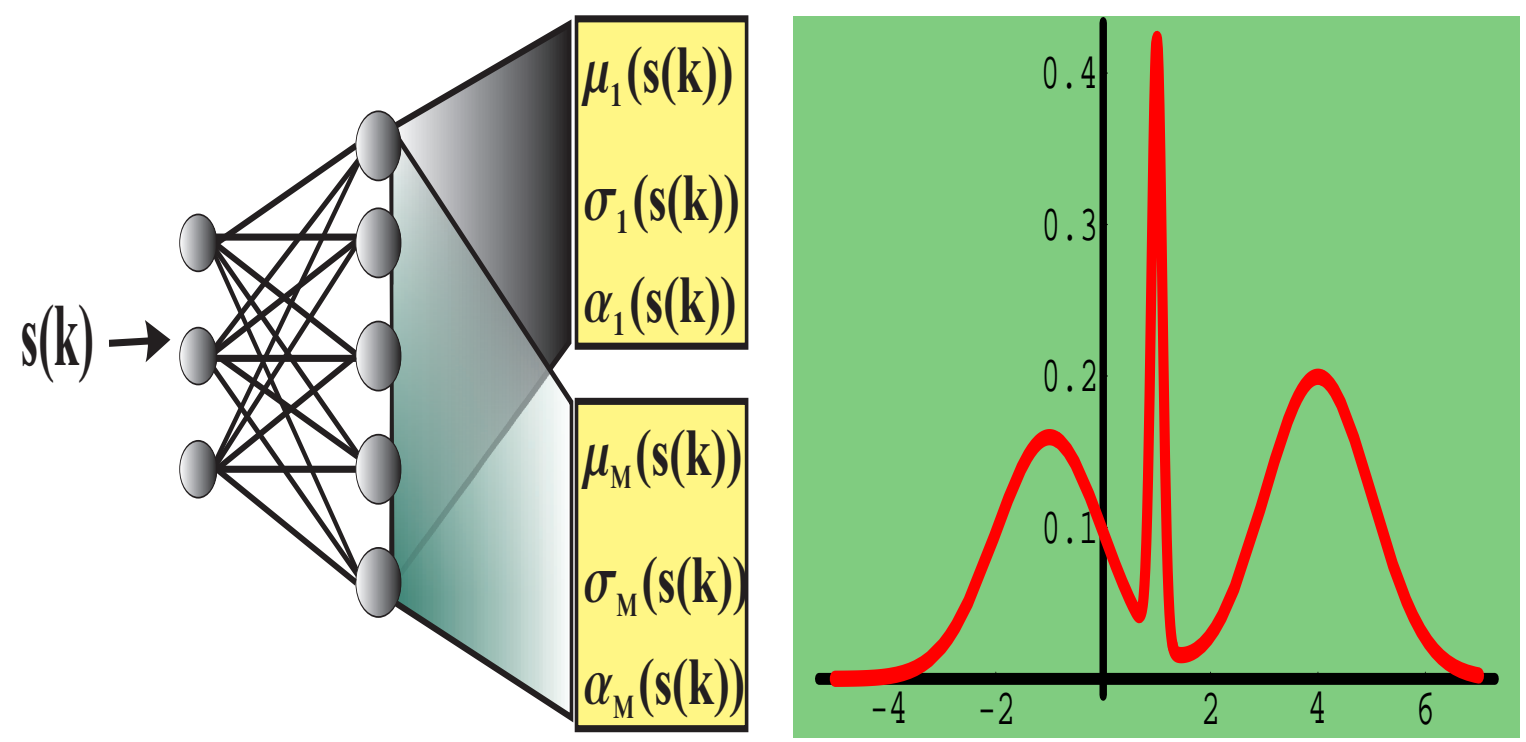

Fig. 9. The architecture of the mixture density network. The inputs to the model $s(k)=\left[y_{d}(k+d), x(k)\right]$ and the outputs estimate the parameters of the mixture components.

Consequently Herzallah [17] proposed an inversion based neuro-controller for incorporating uncertainty in the mixture density network. Similar to the discussion in Section III-A the approach is based on importance sampling, this time from the non-Gaussian distribution of the inverse controller. The optimal control signal is searched for by generating samples from that distribution which are then forwarded to the plant model. The optimal control signal is then taken to be the one that minimizes a performance index of the form given in (1).

In contrast to selecting a specific quantity to represent the output of the mixture density network, the approach in [17] has the advantage of considering the full distribution which is estimated using a mixture density network.

The validity of the proposed method in [17] is demonstrated on a third order system with two 
inputs and two outputs described by the following state equation :

$$
\begin{aligned}
x_{1}(k+1) & =0.9 x_{1}(k) \sin \left[x_{2}(k)\right]+\left[2+1.5 \frac{x_{1}(k) u_{1}(k)}{1+x_{1}^{2}(k) u_{1}^{2}(k)}\right] u_{1}(k)+\left[x_{1}(k)+\frac{2 x_{1}(k)}{1+x_{1}^{2}(k)}\right] u_{2}(k) \\
x_{2}(k+1) & =x_{3}(k)\left\{1+\sin \left[4 x_{3}(k)\right]\right\}+\frac{x_{3}(k)}{1+x_{3}^{2}(k)} \\
x_{3}(k+1) & =\left\{3+\sin \left[2 x_{1}(k)\right]\right\} u_{2}(k) \\
y_{1}(k) & =x_{1}(k) \\
y_{2}(k) & =x_{2}(k)
\end{aligned}
$$

where $\boldsymbol{x}(k)=\left[x_{1}(k), x_{2}(k), x_{3}(k)\right]$ is the state, $\mathbf{u}(k)=\left[u_{1}(k), u_{2}(k)\right]$ is the control variable, and $\mathbf{y}(\mathrm{k})=\left[\mathrm{y}_{1}(\mathrm{k}), \mathrm{y}_{2}(\mathrm{k})\right]$ is the output. Figures $10,11,12$ show the results obtained from using the most probable value, the most likely value of the mixture density network as control signal and the control results from sampling the mixture density network respectively. From these figures it is clear that the performance of the controller by sampling the mixture density network has the best performance.

\section{OPEN PROBLEMS AND FUtURE WORK}

The discussion in this paper focused on incorporating functional uncertainty in three control methods: direct inverse control, indirect adaptive control and the adaptive critic. Incorporating model uncertainty in other control methods has also been considered in the control literature [7], [8], [22], [23], [25], [38].

Although some progress has been achieved for incorporating model uncertainty in the intelligent control framework, the problem has not been completely solved. Almost all the methods discussed in this paper were limited to considering model uncertainty which results because of uncertain model parameters or uncertain model outputs. Functional uncertainty which could result because of the wrong order of the model, the wrong structure or even because of missing data has not been considered.

In addition, the use of the mixture density network has been rarely reported in the control literature. This network has been demonstrated to give good results when used as a controller [17], and it also can model the conditional distribution of the controller or the forward model. The use of this network needs further investigation; such as the possibility of using this network to 


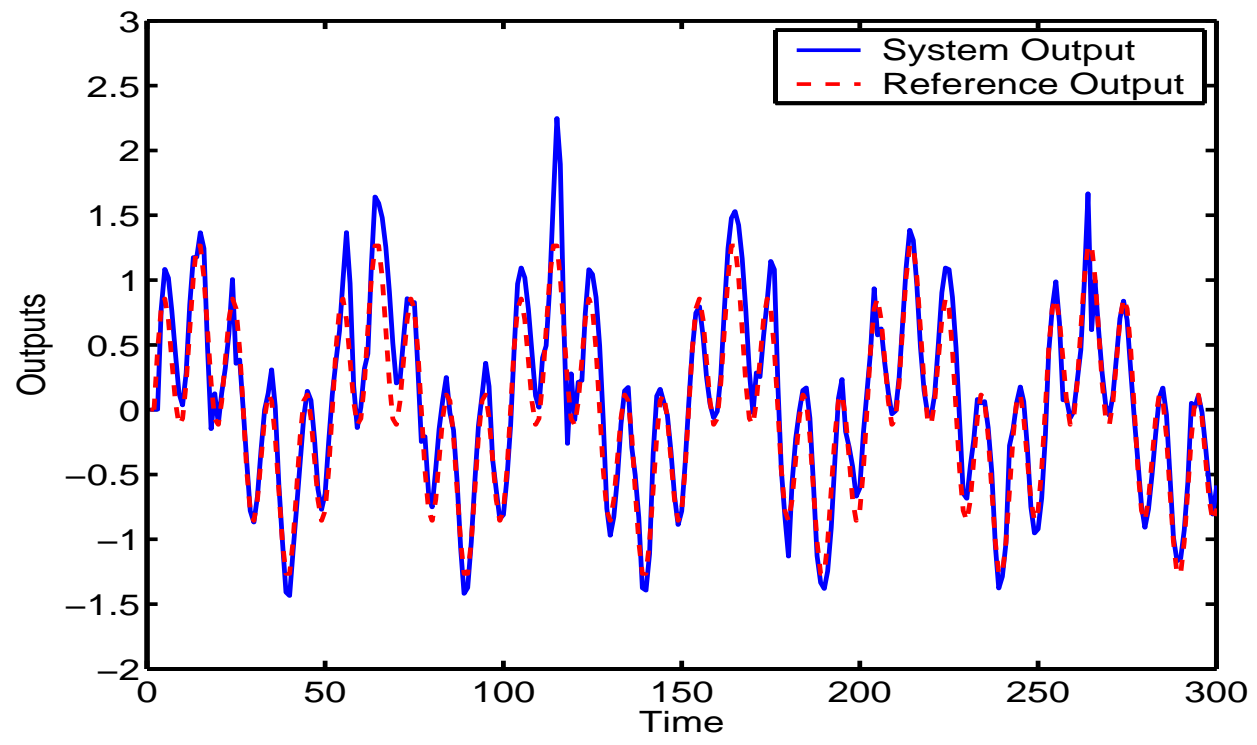

(a)

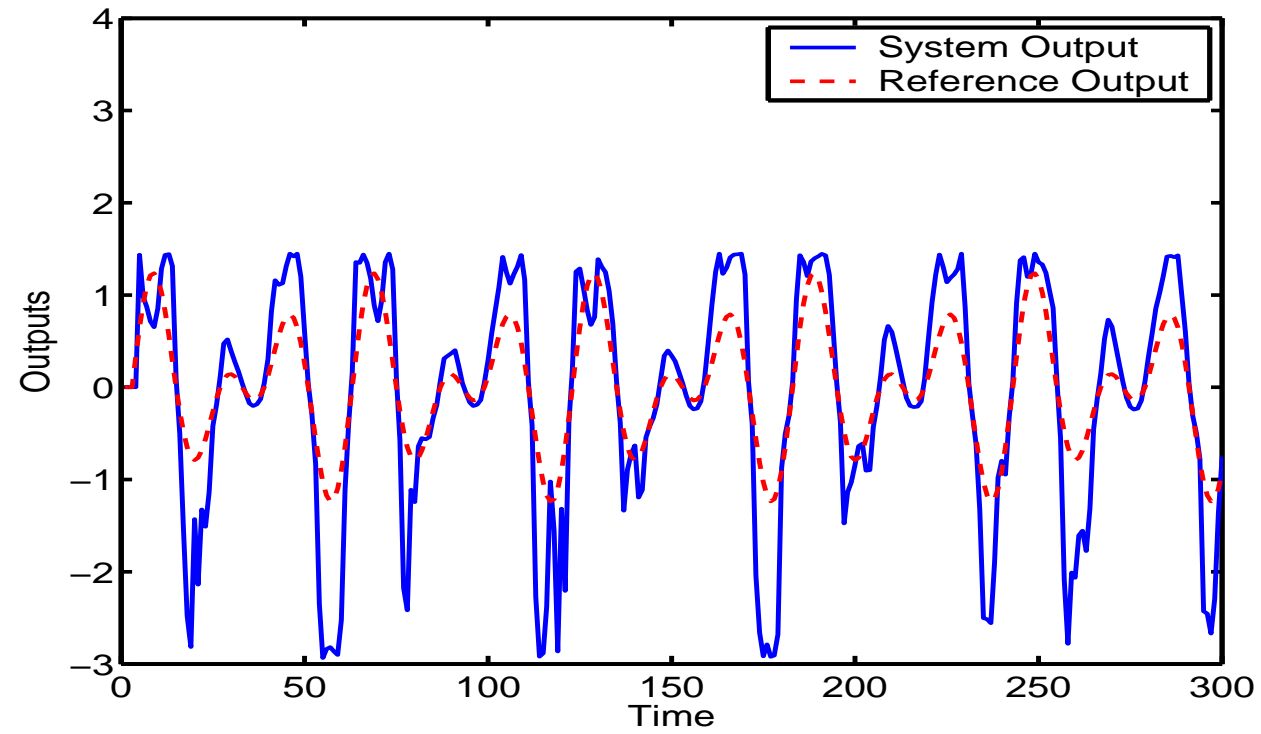

(b)

Fig. 10. Performance of the most probable control value of the mixture density network : (a) the first output of the plant. (b) the second output of the plant. Note the large overshoots in the second output. 


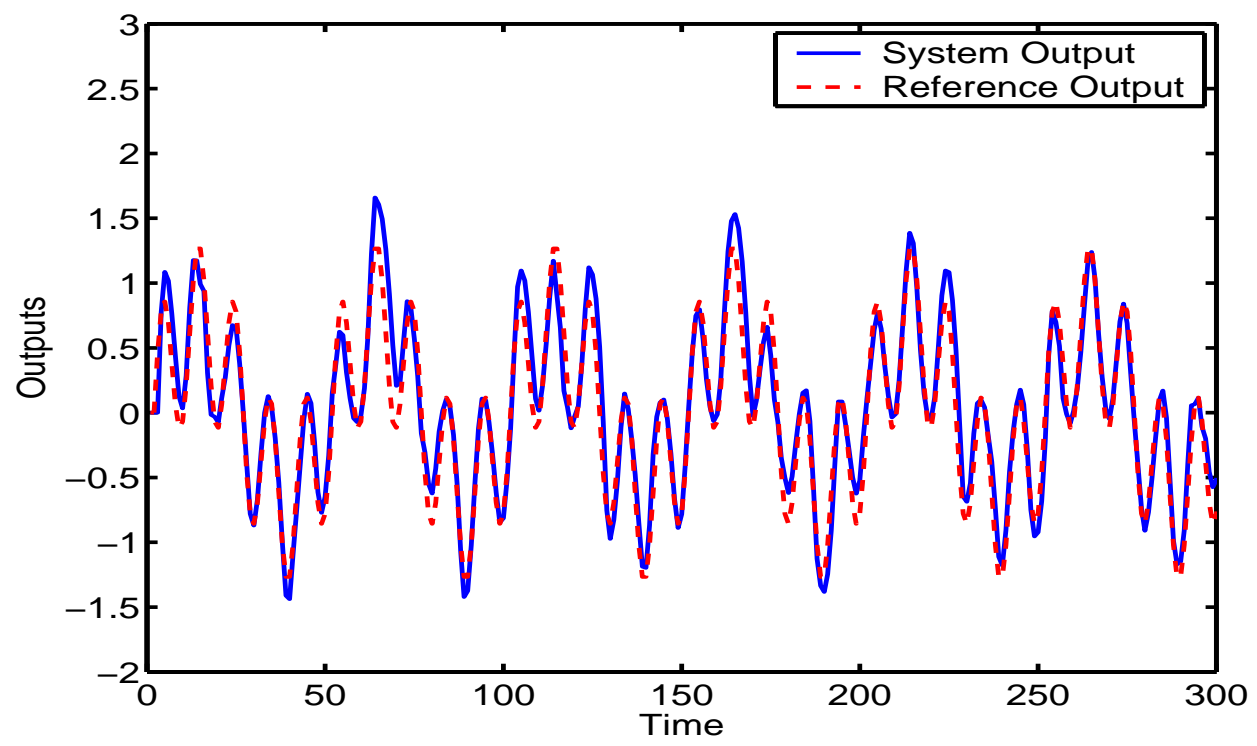

(a)

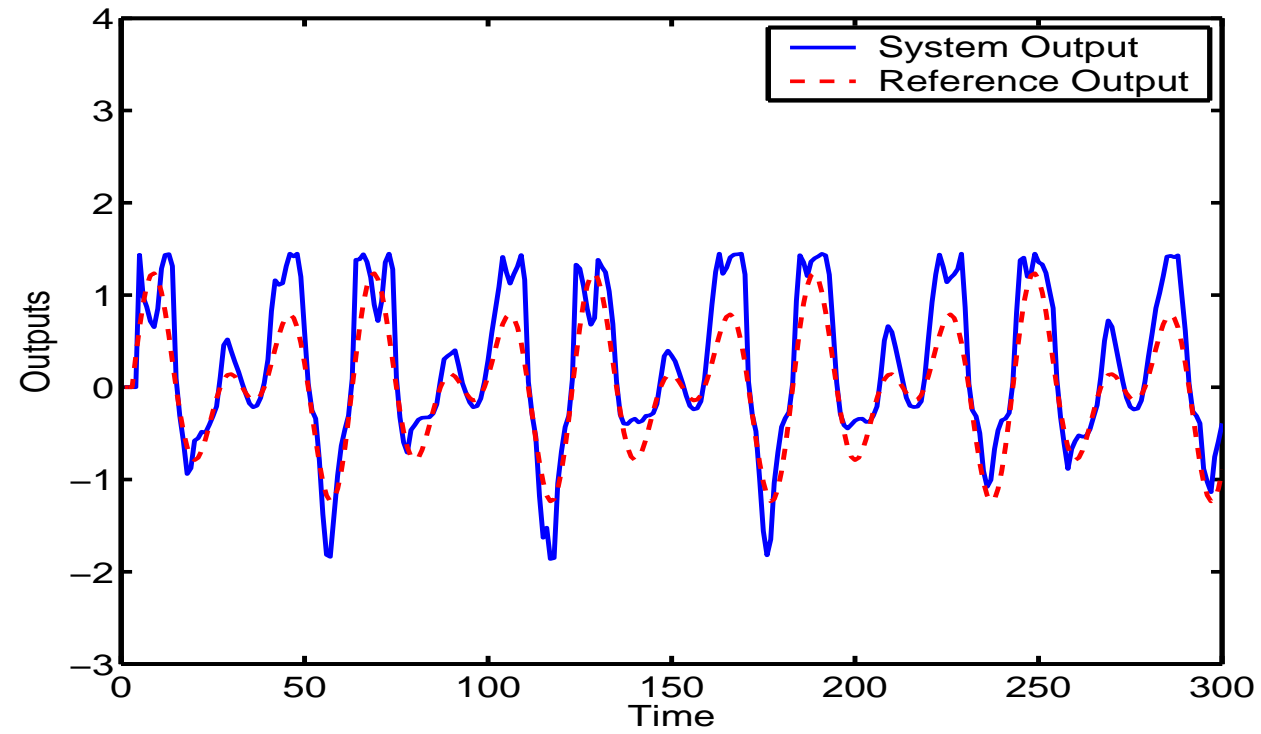

(b)

Fig. 11. Performance of the most likely value of the mixture density network: (a) the first output of the plant. (b) the second output of the plant. The overshoots in the second output have been reduced. 


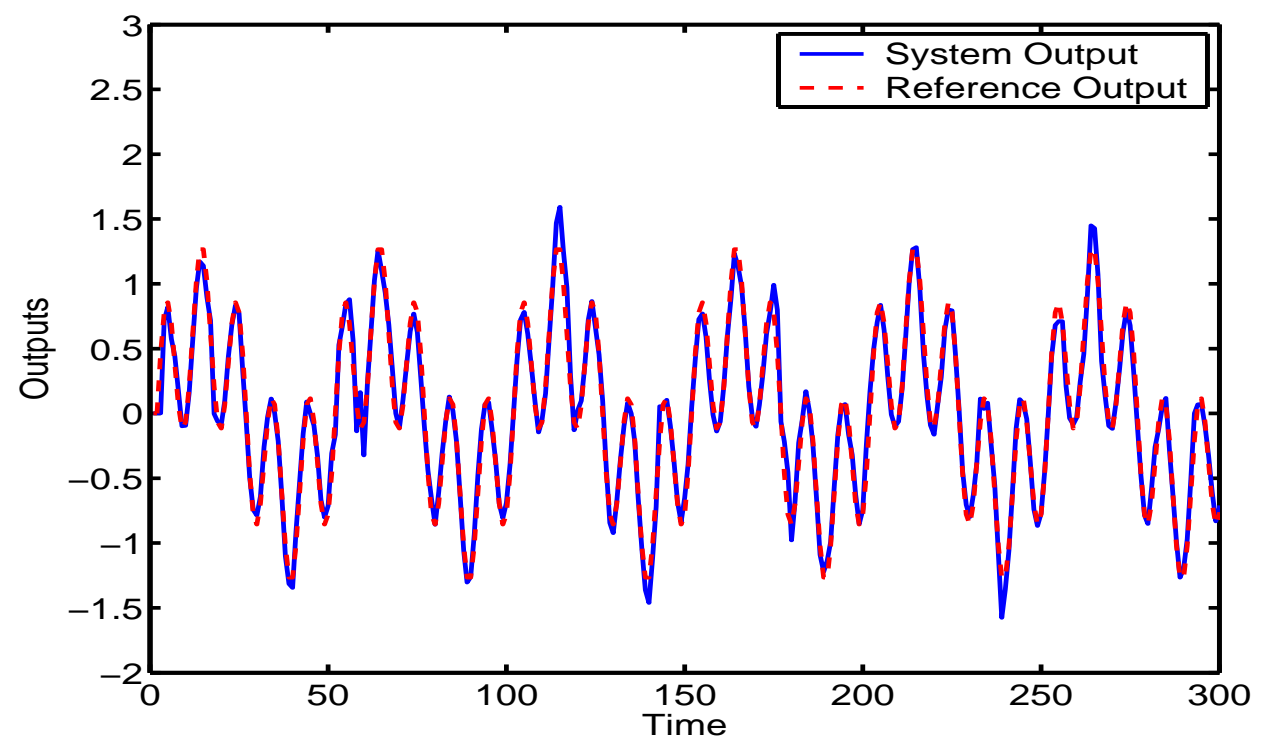

(a)

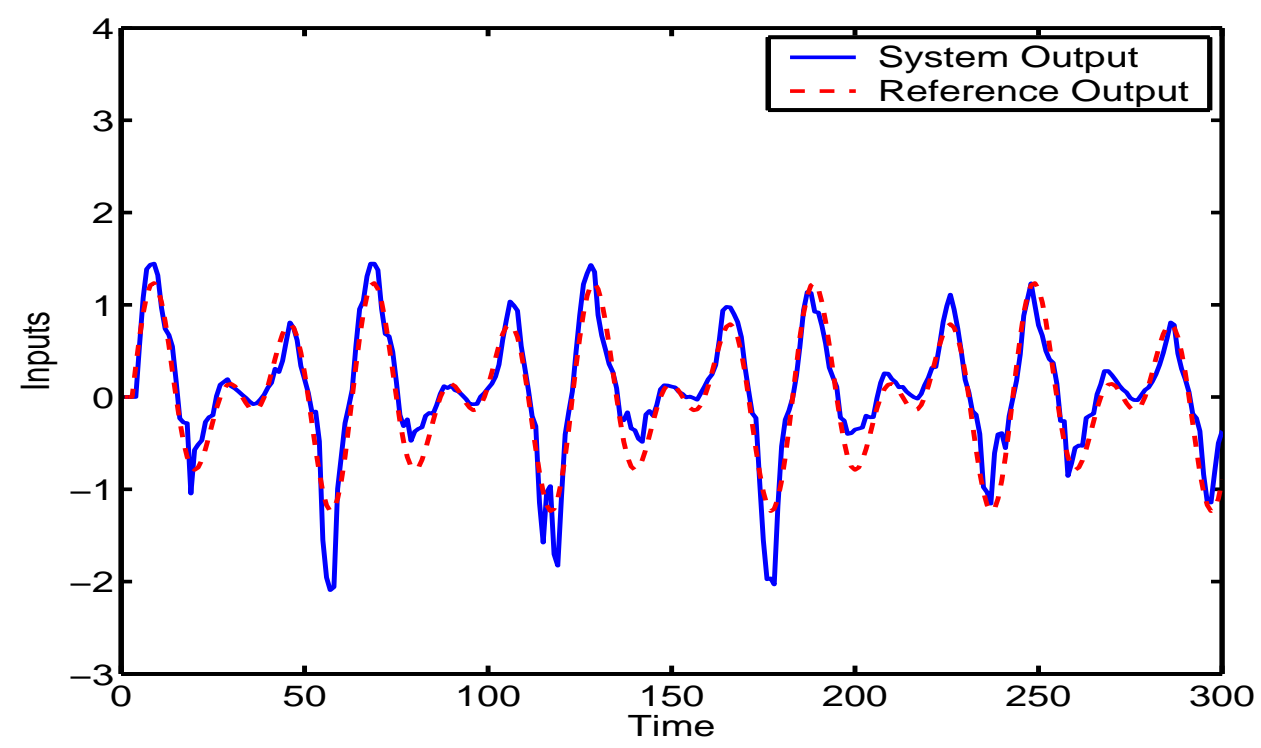

(b)

Fig. 12. Performance of the proposed control approach of mixture density network for the dynamical MIMO system: (a) the first output of the plant. (b) the second output of the plant. The overshoots have been reduced and the average error on the first output is also reduced compared to the previous two methods for selecting a control signal. 
model forward and inverse models in the indirect adaptive control framework or even in the adaptive critic methods.

\section{CONCLUSIONS}

This paper has provided a survey of some of the recently developed methods in the neural control field for incorporating model uncertainty. The basic ideas, the strength and the weakness of each method, and relations with conventional methods have been summarized. The methods discussed in the paper are mainly based on utilizing statistical techniques for modeling the conditional distributions of the outputs or parameters of the neural networks. We explored advantages and disadvantages of each method and discussed the links between the different methods in a unified presentation and identified key areas for future research.

This survey is aimed at researchers currently working in the control field. By putting together some of the publications related to incorporating uncertainty in control problems, we hope that interested researchers may find out about the current status of this field.

\section{REFERENCES}

[1] J. S. Albus. A new approach to manipulator control: The cerebellar model articulation controller (CMAC). Transactions of the ASME Journal of Dynamic Systems, Measurements, and Control, 97:220-227, 1975.

[2] K. J. Åström and B. Wittenmark. Adaptive Control. Addison-Wesley, Reading, MA, U.S.A, 1989.

[3] S. N. Balakrishnan and V. Biega. Adaptive-critic-based neural networks for artificial optimal control. Journal of Guidance, Control, and Dynamics, 19(4):893-898, July-August 1996.

[4] D. R. Baughman and Y. A. Liu. Neural Networks in Bioprocessing and Chemical Engineering. Academic Press, Inc, 1995.

[5] R. E. Bellman. Applied Dynamic Programming. Princeton University Press, Princeton, NJ, 1962.

[6] C. M. Bishop. Neural Networks for Pattern Recognition. Oxford University Press, New York, N.Y., 1995.

[7] M. A. Botto, B. Wams, van den Boom, and J. M. G. Sà da Costa. Robust stability of feedback linearised systems modelled with neural networks: Dealing with uncertainty. Engineering Applications of Artificial Intelligence, 13(6):659-670, 2000.

[8] M. A. Botto, B. Wams, van den Boom, and J. M. G. Sà da Costa. Stability analysis of nonlinear plants under approximate feedback linearization. In J. Maciejowski (eds.); CONTROL 2000 proceedings. UKACC International Conference on Control, pages 1-6, Cambridge, UK, September 2000. project code: ET00-06.

[9] F. C. Chen and C. C. Liu. Adaptively controlling nonlinear continuous-time systems using multilayer neural networks. IEEE Transactions on Automatic Control, 39(6):1306-1310, June 1994.

[10] S. G. Fabri and V. Kadirkamanathan. Functional Adaptive Control: An Intelligent Systems Approach. Springer-Verlag, February 2001.

[11] A. A. Fel'dbaum. Dual control theory i-ii. Automation and Remote Control, 21:874-880, 1033-1039, 1960.

[12] A. A. Fel'dbaum. Dual control theory iii-iv. Automation and Remote Control, 22:1-12, 109-121, 1961. 
[13] A. A. Fel'dbaum. Optimal Control Systems. Academic Press, New York, 1965.

[14] A. Gersho and R.M. Gray. Vector Quantization and Signal Compression. Kluwer Academic Publishers, 1992.

[15] G. C. Goodwin and K. S. Sin. Adaptive Filtering, Prediction and Control. Prentice-Hall, Englewood Cliffs, New Jersy, 1984.

[16] J. A. Gustafson and P. S. Maybeck. Flexible spacestructure control via moving-bank multiple model algorithms. IEEE Transactions on Aerospace and Electronic Systems, 30(3):750-757, July 1994.

[17] R. Herzallah. Exploiting Uncertainty in Nonlinear Stochastic Control Problems. Phd, Aston University, November 2003.

[18] R. Herzallah. Adaptive critic methods for stochastic systems with input-dependent noise. Automatica, 43(8):1355-1362, 2007.

[19] R. Herzallah. Dual version of neural adaptive controller for stochastic nonlinear systems with functional uncertainty. Journal of Control and Intelligent Systems, 35(4):329-336, 2007.

[20] R. Herzallah and D. Lowe. A bayesian approach to modeling the conditional density of the inverse controller. In IEEE Conference on Control Applications, CCA, volume 2, pages 954-959, Taipei, Taiwan, September 2004.

[21] R. Herzallah and D. Lowe. A mixture density network approach to modelling and exploiting uncertainty in nonlinear control problems. Engineering Applications of Artificial Intelligence, 17:145-158, February 2004.

[22] N. Hovakimyan and A. J. Calise. Adaptive output feedback control of uncertain multi-input multi-output systems using single hidden layer neural networks. In Proceedings of the American Control Conference, volume 2, pages 1555-1560, Anchorage, Alaska, USA, May 2002.

[23] N. Hovakimyan, F. Nardi, and A. J. Calise. A novel observer based adaptive output feedback approach for control of uncertain systems. In Proceedings of the American Control Conference, volume 3, pages 2444-2449, Arlington, VA, USA, June 2001.

[24] R. A. Howard. Dynamic Programming and Markov Processes. The Massachusetts Institute of Technology and John Wiley and Sons, Inc., New York. London, 1960.

[25] Z. Huang and S. N. Balakrishnan. Robust adaptive critic based neurocontrollers for systems with input uncertainties. In IEEE International Joint Conference on Neural Networks - IJCNN'O0, volume 3, pages 3067-3072, Como, Italy, 24-27 July 2000.

[26] K. J. Hunt and D. Sbarbaro. Neural networks for nonlinear internal model control. IEE Proceedings-D, 138(5):431-438, September 1991.

[27] K. J. Hunt, D. Sbarbaro, R. Zbikowski, and P. J. Gawthrop. Neural networks for control systems-a survey. Automatica, 28(6):1083-1112, 1992.

[28] A. Isidori. Nonlinear Control Systems, An Introduction. Springer-Verlag, Berlin, Heidelberg, 1989.

[29] R. A. Jacobs and S. J. Nowlan. Adaptive mixtures of local experts. Neural Computation, 3:79-87, 1991.

[30] T. A. Johansen and B. A. Foss. Identification of non-linear system structure and parameters using regime decomposition. Automatica, 31(2):321-326, 1995.

[31] M. I. Jordan and R. A. Jacobs. Hierarchical mixtures of experts and the EM algorithm. Neural Computation, 6:181-214, 1994.

[32] M. I. Jordan and L. Xu. Convergence results for the EM approach to mixtures of experts architectures. Technical report, A.I. Memo no.1458, C.B.C.L. Memo No. 87, Massachusetts Institute of Technology, Artificial Intelligence Laboratory and Center of Biological and Computational Learning Department of Brain and Cognitive Sciences, November 181993. 
[33] M. Krstic, I. Kanellakopoulos, and P. Kokotovic. Nonlinear and Adaptive Control Design. Wiley Series on Adaptive and Learning Systems for Signal processing, Communications, and Control. Wiley-Interscience, U.S.A, 1995.

[34] D. G. Lainiotis. Partitioninig: A unifying framework for adaptive systems, 1: Estimation. Proceedings of the IEEE, 64(8):1126-1143, August 1976.

[35] R. E. Larson. State Increment Dynamic Programming. Number 12 in Modern Analytical and Computational Methods in Science and Mathematics. American Elsevier Publishing Company, New York, N.Y, 1968.

[36] G. Lightbody and G. W. Irwin. Nonlinear control structures based on embedded neural system models. IEEE Transactions on Neural Networks, 8(3):553-567, May 1997.

[37] P. S. Maybeck and R. D. Stevens. Reconfigurable flight control via multiple model adaptive control methods. IEEE Transactions on Aerospace and Electronic Systems, 27(3):470-480, May 1991.

[38] M. B. McFarland and A. J. Calise. Robust adaptive control of uncertain nonlinear systems using neural networks. In Proceedings of the American Control Conference, volume 3, pages 1996-2000, June 1997.

[39] W. T. Miller, R. S. Sutton, and P. J. Werbos, editors. Neural Networks for Control. MIT Press, Cambridge, Massachusetts, London, England., 1990.

[40] L. Moreno, L. Acosta, J. A. Méndez, A. Hamilton, G. N. Marichal, J. D. Pñeiro, and J. L. Sánchez. Stochastic optimal controllers for a DC servo motor: Applicability and performance. Control Engineering Practice, 4(6):757-764, 1996.

[41] M.Polycarpou and P. Ioannou. Identification and control of nonlinear systems ssing neural network models: Design and stability analysis. Technical report 91-09-01, Department of Electrical Engineering Systems, University of Southern California, Los Angeles, USA, September 1991.

[42] K. S. Narendra. Neural networks for control: Theory and practice. Proceedings of the IEEE, 84(10):1385-1406, October 1996.

[43] K. S. Narendra and A. M. Annaswamy. Stable Adaptive Systems. Prentice-Hall, Englewood Cliffs, New Jersey, 1989.

[44] K. S. Narendra and J. Balakrishnan. Improving transient response of adaptive control systems using multiple models and switching. IEEE Transactions on Automatic Control, 39(9):1861-1866, September 1994.

[45] K. S. Narendra and J. Balakrishnan. Adaptive control using multiple models. IEEE Transactions on Automatic Control, 42(2):171-187, February 1997.

[46] K. S. Narendra, J. Balakrishnan, and M. K. Ciliz. Adaptation and learning using multiple models, switching, and tuning. IEEE Control Systems Magazine, 15:37-51, June 1995.

[47] K. S. Narendra, R. Ortega, and P. Dorato, editors. Advances in Adaptive Control. IEEE Press, U.S.A, 1991.

[48] K. S. Narendra and K. Parthasarathy. Identification and control of dynamical systems using neural networks. IEEE Transactions on Neural Networks, 1:4-26, 1990.

[49] D. V. Prokhorov and D. C. Wunsch. Adaptive critic designs. IEEE Transactions on Neural Networks, 8(5):997-1007, September 1997.

[50] D. Psaltis, A. Sideris, and A. A. Yamamura. A multilayered neural network controller. IEEE Control Systems Magazine, 88(2):17-21, 1988.

[51] H. E. Rauch. Autonomous control reconfiguration. IEEE Control Systems Magazine, 15:37-48, December 1995.

[52] W. J. Runggaldier. Concepts and methods for discrete and continuous time control under uncertainty. Insurance: Mathematics and Economics, 22:25-39, 1998.

[53] R. M. Sanner and J. E. Soltine. Gaussian networks for direct adaptive control. IEEE Transactions on Neural Networks, 3(6):837-863, 1992. 
[54] S. S. Sastry and A. Isidori. Adaptive control of linearizable systems. IEEE Transactions on Automatic Control, 34:11231131, 1989.

[55] E. Tzirkel-Hancock and F. Fallside. Stable control of nonlinear systems using neural networks. International Journal of Robust and Nonlinear Control, 2:63-86, May 1992.

[56] P. J. Werbos. Approximate dynamic programming for real-time control and neural modeling. In D. A. White and D. A. Sofge, editors, Handbook of Intelligent Control, pages 493-525. Multiscience Press, Inc, New York, N.Y., 1992.

[57] D. A. White and D. Sofge, editors. Handbook of Intelligent Control. Multiscience Press, Inc, New York, N.Y., 1992.

[58] B. Wittenmark. Stochastic adaptive control methods: A survey. International Journal of Control, 21:705-730, 1975.

[59] D. M. Wolpert and M. Kawato. Multiple paired forward and inverse models for motor control. Neural Networks, 11:13171329, 1998.

[60] P. C. Yeh and P. V. Kokotovic. Adaptive output-feedback design for a class of nonlinear discrete time systems. IEEE Transactions on Automatic Control, 40:1663-1668, 1995.

[61] A. Yesildirek and F. L. Lewis. Feedback linearization using neural networks. Automatica, 31(11):1659-1664, 1995. 